\title{
Existence, Uniqueness, and Characterization Theorems for Nonlinear Fuzzy Integrodifferential Equations of Volterra Type
}

\author{
Omar Abu Arqub, ${ }^{1}$ Shaher Momani, ${ }^{2,3}$ Saleh Al-Mezel, ${ }^{3}$ and Marwan Kutbi ${ }^{3}$ \\ ${ }^{1}$ Department of Mathematics, Faculty of Science, Al Balqa Applied University, Salt 19117, Jordan \\ ${ }^{2}$ Department of Mathematics, Faculty of Science, The University of Jordan, Amman 11942, Jordan \\ ${ }^{3}$ Nonlinear Analysis and Applied Mathematics (NAAM) Research Group, Department of Mathematics, Faculty of Science, \\ King Abdulaziz University (KAU), Jeddah 21589, Saudi Arabia \\ Correspondence should be addressed to Omar Abu Arqub; o.abuarqub@bau.edu.jo
}

Received 12 October 2014; Accepted 18 November 2014

Academic Editor: Jinhu Lü

Copyright (C) 2015 Omar Abu Arqub et al. This is an open access article distributed under the Creative Commons Attribution License, which permits unrestricted use, distribution, and reproduction in any medium, provided the original work is properly cited.

\begin{abstract}
Existence and uniqueness theorem are the tool which makes it possible for us to conclude that there exists only one solution to a given problem which satisfies a constraint condition. How does it work? Why is it the case? We believe it, but it would be interesting to see the main ideas behind this. To this end, in this paper, we investigate existence, uniqueness, and other properties of solutions of a certain nonlinear fuzzy Volterra integrodifferential equation under strongly generalized differentiability. The main tools employed in the analysis are based on the applications of the Banach fixed point theorem and a certain integral inequality with explicit estimate. Also, some results for characterizing solution by an equivalent system of crisp Volterra integrodifferential equations are presented. In this way, a new direction for the methods of analytic and approximate solutions is proposed.
\end{abstract}

\section{Introduction}

Many important real-world problems of analytical dynamics are described by the nonlinear mathematical models that, as a rule, are presented and modeled by the nonlinear crisp (ordinary) integrodifferential equations (IDEs). Usually, we cannot be sure that this modeling is perfect, because, in many situations, information about the real-world phenomena involved is always pervaded with uncertainty. The uncertainty can arise in experiment part, data collection, and measurement process as well as when determining the constraints conditions. Therefore, it is necessary to have some mathematical apparatus and tools in order to understand this uncertainty. In fact, the aforementioned factors will lead to errors; if the nature of errors is random, then we get a random IDE with random constraints conditions and/or random coefficients. But if the underlying structure is not probabilistic, because of subjective choice, then it may be appropriate to use fuzzy IDE with fuzzy constraints conditions and/or fuzzy coefficients. Anyhow, fuzzy IDEs are utilized to analyze the behavior of phenomena that are subject to imprecise or uncertain factors.
The study of fuzzy IDEs has gained importance in recent times; here, we are focusing our attention on first-order fuzzy Volterra IDEs (VIDEs) subject to given fuzzy initial condition. At the beginning, approaches to fuzzy IDEs and other fuzzy equations can be of three types. The first approach assumes that even if only the initial value is fuzzy, the solution is a fuzzy function, and, consequently, the derivatives in the IDE must be considered as fuzzy derivatives $[1,2]$. These can be done by the use of the Hukuhara derivative for fuzzyvalued functions. Generally, this approach has a drawback; the solution becomes fuzzier as time goes; hence, the fuzzy solution behaves quite differently from the crisp solution. In the second approach, the fuzzy IDE is transformed to a crisp one by interpreting it as a family of differential inclusions $[3,4]$. The main shortcoming of using differential inclusions is that we do not have a derivative of a fuzzy-valued function. The third approach is based on the Zadeh's extension principle, where the associated crisp problem is solved and in the solution the initial fuzzy values are substituted instead of the real constants, and, in the final solution, arithmetic operations are considered to be operations on fuzzy numbers $[5,6]$. The weakness of this approach is the need to rewrite 
the solution in the fuzzy setting which in turn makes the methods of solution are not user-friendly and more restricted with more computation steps. As a conclusion, to overcome the above-mentioned shortcoming, the concept of a strongly generalized differentiability was developed and investigated in [7-14]. Anyhow, using the strongly generalized differentiability, the fuzzy IDE has locally two solutions. Indeed, with this approach, we can find solutions for a larger class of fuzzy IDEs compared to using other types of differentiability.

Bear in mind that not every fuzzy VIDE is solvable. But that does not mean that a solution does not exist. This is a mathematical subtlety that may not be obvious at first. There is a large divide in math between knowing that something exists and actually constructing it. In fact, we must come to grips with this idea if we are to understand the motivation for the existence and uniqueness theorem. Anyhow, it is worth stating that, in many cases, since fuzzy VIDEs are often derived from problems in physical world, existence and uniqueness are often obvious for physical reasons. Notwithstanding this, a mathematical statement about existence and uniqueness is worthwhile. Uniqueness would be of importance if, for instance, we wished to approximate the solutions. If two solutions passed through a point, then successive approximations could very well jump from one solution to the other with misleading consequences.

The purpose of this paper is to investigate the characterization theorem together with the existence and unicity of two solutions, one solution for each lateral derivative, to firstorder fuzzy IDEs of Volterra type under the assumption of strongly generalized differentiability of the general form:

$$
x^{\prime}(t)=f(t, x(t))+\int_{t_{0}}^{t} g(t, \tau, x(\tau)) d \tau, \quad t_{0} \leq t, \tau \leq t_{0}+a,
$$

subject to the fuzzy initial condition

$$
x\left(t_{0}\right)=\sigma,
$$

where $f:\left[t_{0}, t_{0}+a\right] \times \mathbb{R}_{\mathscr{F}} \rightarrow \mathbb{R}_{\mathscr{F}}$ and $g:\left[t_{0}, t_{0}+\right.$ $a]^{2} \times \mathbb{R}_{\mathscr{F}} \rightarrow \mathbb{R}_{\mathscr{F}}$ are continuous fuzzy-valued functions that satisfy a Lipchitz condition, $\sigma \in \mathbb{R}_{\mathscr{F}}$, and $t_{0}$, $a$ are real finite constants with $a>0$.

The solvability theory of fuzzy VIDEs has been studied by several researchers by using the strongly generalized differentiability, the Hukuhara derivative, or the Zadeh's extension principle for the fuzzy-valued mappings of a real variable whose values are normal, convex, upper semicontinuous, and compactly supported fuzzy sets in $\mathbb{R}$. The reader is asked to refer to [15-22] in order to know more details about these analyses, including their kinds and history, their modifications and conditions for use, their scientific applications, their importance and characteristics, and their relationship including the differences. But, on the other aspect as well, more details about the solvability theory of crisp VIDEs can be found in $[23,24]$ and more details about the characterization theorem can be found in $[25,26]$.

The organization of the paper is as follows. In the next section, we present some necessary definitions and preliminary results from the fuzzy calculus theory. The procedure of solving fuzzy VIDEs is presented in Section 3. In Section 4, existence and uniqueness of two solutions are introduced. In Section 5, we utilize two characterization theorems and some properties for the solution of fuzzy VIDEs. This paper ends at Section 6 with some concluding remarks.

\section{Background Material of Fuzzy Calculus Theory}

The backward of theory of fuzzy VIDEs extremely appears in the references [15-22] side by side with their applications in the field of engineering problems, applied mathematics, theoretical physics, and mathematical finance. Anyhow, for the reader's convenience, we present some necessary definitions from fuzzy calculus theory and preliminary results. For the concept of fuzzy derivative, we will adopt strongly generalized differentiability, which is a modification of the Hukuhara differentiability and has the advantage of dealing properly with fuzzy VIDEs.

Let $X$ be a nonempty set. A fuzzy set $u$ in $X$ is characterized by its membership function $u: X \rightarrow[0,1]$. Thus, $u(s)$ is interpreted as the degree of membership of an element $s$ in the fuzzy set $u$ for each $s \in X$. A fuzzy set $u$ on $\mathbb{R}$ is called convex if for each $s, t \in \mathbb{R}$ and $\lambda \in[0,1], u(\lambda s+(1-\lambda) t) \geq$ $\min \{u(s), u(t)\}$, is called upper semicontinuous if $\{s \in \mathbb{R}:$ $u(s)>r\}$ is closed for each $r \in[0,1]$, and is called normal if there is $s \in \mathbb{R}$ such that $u(s)=1$. The support of a fuzzy set $u$ is defined as $\{s \in \mathbb{R}: u(s)>0\}$.

Definition 1 (see [27]). A fuzzy number $u$ is a fuzzy subset of the real line with a normal, convex, and upper semicontinuous membership function of bounded support.

For each $r \in(0,1]$, set $[u]^{r}=\{s \in \mathbb{R}: u(s) \geq r\}$ and $[u]^{0}=$ $\overline{\{s \in \mathbb{R}: u(s)>0\}}$, where $\overline{\{\cdot\}}$ denotes the closure of $\{\cdot\}$. Then, it easy to establish that $u$ is a fuzzy number if and only if $[u]^{r}$ is compact convex subset of $\mathbb{R}$ for each $r=[0,1]$ and $[u]^{1} \neq \phi$ [28]. Thus, if $u$ is a fuzzy number, then $[u]^{r}=[\underline{u}(r), \bar{u}(r)]$, where $\underline{u}(r)=\min \left\{s: s \in[u]^{r}\right\}$ and $\bar{u}(r)=\max \{s: s \in$ $\left.[u]^{r}\right\}$ for each $r \in[0,1]$. The symbol $[u]^{r}$ is called the $r$-cut representation or parametric form of a fuzzy number $u$. We will let $\mathbb{R}_{\mathscr{F}}$ denote the set of fuzzy numbers on $\mathbb{R}$.

The question arising here is as follows: if we have an interval-valued function $[\underline{z}(r), \bar{z}(r)]$ defined on $[0,1]$, then is there a fuzzy number $u$ such that $[u(r)]^{r}=[\underline{z}(r), \bar{z}(r)]$ ? The next theorem characterizes fuzzy numbers through their $r$ cut representations.

Theorem 2 (see [28]). Suppose that $\underline{u}:[0,1] \rightarrow \mathbb{R}$ and $\bar{u}:[0,1] \rightarrow \mathbb{R}$ satisfy the following conditions: first, $\underline{u}$ is a bounded increasing function and $\bar{u}$ is a bounded decreasing function with $\underline{u}(1) \leq \bar{u}(1)$; second, for each $k \in(0,1], \underline{u}$ and $\bar{u}$ are left-hand continuous functions at $r=k$; third, $\underline{u}$ and $\bar{u}$ are right-hand continuous functions at $r=0$. Then, $u: \mathbb{R} \rightarrow[0,1]$, defined by

$$
u(s)=\sup \{r: \underline{u}(r) \leq s \leq \bar{u}(r)\},
$$

is a fuzzy number with parameterization given by $[\underline{u}(r), \bar{u}(r)]$. Furthermore, if $u: \mathbb{R} \rightarrow[0,1]$ is a fuzzy number with 
parameterization $[\underline{u}(r), \bar{u}(r)]$, then the functions $\underline{u}$ and $\bar{u}$ satisfy the aforementioned conditions.

In general, we can represent an arbitrary fuzzy number $u$ by an order pair of functions $(\underline{u}, \bar{u})$ which satisfy the requirements of Theorem 2 . Frequently, we will write simply $u_{r}$ and $\bar{u}_{r}$ instead of $u(r)$ and $\bar{u}(r)$, respectively.

The metric structure on $\mathbb{R}_{\mathscr{F}}$ is given by $d_{\infty}: \mathbb{R}_{\mathscr{F}} \times \mathbb{R}_{\mathscr{F}} \rightarrow$ $\mathbb{R}^{+} \cup\{0\}$ such that $d_{\infty}(u, v)=\sup _{r \in[0,1]} d_{H}\left([u]^{r},[v]^{r}\right)$ for arbitrary fuzzy numbers $u$ and $v$, where $d_{H}$ is the Hausdorff metric between $[u]^{r}$ and $[v]^{r}$. This metric is defined as $d_{H}\left([u]^{r},[v]^{r}\right)=\inf \left\{\varepsilon:[u]^{r} \subset N\left([u]^{r}, \varepsilon\right),[v]^{r} \subset N\left([v]^{r}, \varepsilon\right)\right\}=$ $\max \left\{\left|\underline{u}_{r}-\underline{v}_{r}\right|,\left|\bar{u}_{r}-\bar{v}_{r}\right|\right\}$, where the two sets $N\left([u]^{r}, \varepsilon\right)$ and $N\left([v]^{r}, \varepsilon\right)$ are the $\varepsilon$-neighborhoods of $[u]^{r}$ and $[v]^{r}$, respectively. It is shown in [29] that $\left(\mathbb{R}_{\mathscr{F}}, d_{\infty}\right)$ is a complete metric space.

Lemma 3 (see [29]). For each $A, B, C, D \in \mathbb{R}_{\mathscr{F}}$ with $\lambda \in \mathbb{R}$, the metric function $d_{\infty}$ satisfies the following properties:

(i) $d_{\infty}(A+C, B+C)=d_{\infty}(A, B)$;

(ii) $d_{\infty}(A, B) \leq d_{\infty}(A, C)+d_{\infty}(C, B)$;

(iii) $d_{\infty}(A+C, B+D) \leq d_{\infty}(A, B)+d_{\infty}(C, D)$;

(iv) $d_{\infty}(\lambda A, \lambda B)=|\lambda| d_{\infty}(A, B)$.

For arithmetic operations on fuzzy numbers, the following results are well known and follow from the theory of interval analysis. If $u$ and $v$ are two fuzzy numbers, then, for each $r \in[0,1]$, we have, firstly, $[u+v]^{r}=[u]^{r}+[v]^{r}=$ $\left[\underline{u}_{r}+\underline{v}_{r}, \bar{u}_{r}+\bar{v}_{r}\right]$; secondly, $[\lambda u]^{r}=\lambda[u]^{r}=\left[\min \left\{\lambda \underline{u}_{r}, \lambda \bar{u}_{r}\right\}\right.$, $\left.\max \left\{\lambda \underline{u}_{r}, \lambda \bar{u}_{r}\right\}\right]$; thirdly, $[u v]^{r}=[u]^{r}[v]^{r}=\left[\min \left\{\underline{u}_{r} \underline{v}_{r}, \underline{u}_{r} \bar{v}_{r}\right.\right.$, $\left.\left.\bar{u}_{r} \underline{v}_{r}, \bar{u}_{r} \bar{\nu}_{r}\right\}, \max \left\{\underline{u}_{r} \underline{v}_{r}, \underline{u}_{r} \bar{v}_{r}, \bar{u}_{r} \underline{v}_{r}, \bar{u}_{r} \bar{v}_{r}\right\}\right]$; fourthly, $u=v$ if and only if $[u]^{r}=[v]^{r}$ if and only if $\underline{u}_{r}=\underline{v}_{r}$ and $\bar{u}_{r}=\bar{v}_{r}$. In fact, the collection of all fuzzy numbers with aforementioned addition and scalar multiplication is a convex cone [30].

Let $u, v \in \mathbb{R}_{\mathscr{F}}$. If there exists a $w \in \mathbb{R}_{\mathscr{F}}$ such that $u=$ $v+w$, then $w$ is called the H-difference of $u$ and $v$, denoted by $u \ominus v$. Here, the sign " $\ominus$ " stands always for H-difference and let us remark that $u \ominus v \neq u+(-1) v$. Usually, we denote $u+(-1) v$ by $u-v$, while $u \ominus v$ stands for the H-difference. It follows that Hukuhara differentiable function has increasing length of support [27]. To avoid this difficulty, we consider the following definition.

Definition 4 (see [8]). Let $x:\left[t_{0}, t_{0}+a\right] \rightarrow \mathbb{R}_{\mathscr{F}}$ and $t^{*} \in$ $\left[t_{0}, t_{0}+a\right]$. One says that $x$ is strongly generalized differentiable at $t^{*}$, if there exists an element $x^{\prime}\left(t^{*}\right) \in \mathbb{R}_{\mathscr{F}}$ such that, either

(i) for all $h>0$ being sufficiently close to 0 , the $\mathrm{H}$ differences $x\left(t^{*}+h\right) \ominus x\left(t^{*}\right), x\left(t^{*}\right) \ominus x\left(t^{*}-h\right)$ exist and $\lim _{h \rightarrow 0^{+}}\left(\left(x\left(t^{*}+h\right) \ominus x\left(t^{*}\right)\right) / h\right)=\lim _{h \rightarrow 0^{+}}\left(\left(x\left(t^{*}\right) \ominus\right.\right.$ $\left.\left.x\left(t^{*}-h\right)\right) / h\right)=x^{\prime}\left(t^{*}\right)$;

(ii) for all $h>0$ being sufficiently close to 0 , the $\mathrm{H}$ differences $x\left(t^{*}\right) \ominus x\left(t^{*}+h\right), x\left(t^{*}-h\right) \ominus x\left(t^{*}\right)$ exist and $\lim _{h \rightarrow 0^{+}}\left(\left(x\left(t^{*}\right) \ominus x\left(t^{*}+h\right)\right) /-h\right)=\lim _{h \rightarrow 0^{+}}\left(\left(x\left(t^{*}-\right.\right.\right.$ h) $\left.\left.\ominus x\left(t^{*}\right)\right) /-h\right)=x^{\prime}\left(t^{*}\right)$.

Here, the limit is taken in the metric space $\left(\mathbb{R}_{\mathscr{F}}, d_{\infty}\right)$ and at the endpoints of $\left[t_{0}, t_{0}+a\right]$, we consider only one-sided derivatives. For customizing, in Definition 4, the first case corresponds to the $\mathrm{H}$-derivative introduced in [30], so this differentiability concept is a generalization of the Hukuhara derivative.

Definition 5 (see [10]). Let $x:\left[t_{0}, t_{0}+a\right] \rightarrow \mathbb{R}_{\mathscr{F}}$. One says that $x$ is (1)-differentiable on $\left[t_{0}, t_{0}+a\right]$ if $x$ is differentiable in the sense (i) of Definition 4 and its derivative is denoted by $D_{1} x$. Similarly, one says that $x$ is (2)-differentiable on $\left[t_{0}, t_{0}+\right.$ a] if $x$ is differentiable in the sense (ii) of Definition 4 and its derivative is denoted by $D_{2} x$.

The subsequent theorems show us a way to translate a fuzzy VIDE into a system of crisp VIDEs without the need to consider the fuzzy setting approach. Anyhow, these two theorems have many uses in the applied mathematics and the numerical analysis fields.

Theorem 6 (see [10]). Let $x:\left[t_{0}, t_{0}+a\right] \rightarrow \mathbb{R}_{\mathscr{F}}$ and put $[x(t)]^{r}=\left[\underline{x}_{r}(t), \bar{x}_{r}(t)\right]$ for each $r \in[0,1]$.

(i) If $x$ is (1)-differentiable, then $\underline{x}_{r}$ and $\bar{x}_{r}$ are differentiable functions on $\left[t_{0}, t_{0}+a\right]$ and $\left[D_{1} x(t)\right]^{r}=\left[\underline{x}_{r}^{\prime}(t)\right.$, $\left.\bar{x}_{r}^{\prime}(t)\right]$

(ii) If $x$ is (2)-differentiable, then $\underline{x}_{r}$ and $\bar{x}_{r}$ are differentiable functions on $\left[t_{0}, t_{0}+a\right]$ and $\left[D_{2} x(t)\right]^{r}=\left[\bar{x}_{r}^{\prime}(t)\right.$, $\left.\underline{x}_{r}^{\prime}(t)\right]$.

A fuzzy-valued function $x:\left[t_{0}, t_{0}+a\right] \rightarrow \mathbb{R}_{\mathscr{F}}$ is called continuous at a point $t^{*} \in\left[t_{0}, t_{0}+a\right]$ provided for arbitrary fixed $\varepsilon>0$; there exists an $\delta>0$ such that $d_{\infty}\left(x(t), x\left(t^{*}\right)\right)<\varepsilon$ whenever $\left|t^{*}-t\right|<\delta$ for each $t \in\left[t_{0}, t_{0}+a\right]$. We say that $x$ is continuous on $\left[t_{0}, t_{0}+a\right]$ if $x$ is continuous at each $t^{*} \epsilon$ $\left[t_{0}, t_{0}+a\right]$ such that the continuity is one-sided at endpoints $t_{0}$ and $t_{0}+a$.

In order to complete the expert results about the fuzzy calculus theory, we finalize the present section by some preliminary information about the fuzzy integral. Following [28], we define the integral of a fuzzy-valued function using the Riemann integral concept.

Definition 7 (see [28]). Suppose that $x:\left[t_{0}, t_{0}+a\right] \rightarrow \mathbb{R}_{\mathscr{F}}$, for each partition $\wp=\left\{t_{0}^{*}, t_{1}^{*}, \ldots, t_{n}^{*}\right\}$ of $\left[t_{0}, t_{0}+a\right]$ and for arbitrary points $\xi_{i} \in\left[t_{i-1}^{*}, t_{i}^{*}\right], 1 \leq i \leq n$; let $\mathfrak{R}_{\wp}=$ $\sum_{i=1}^{n} x\left(\xi_{i}\right)\left(t_{i}^{*}-t_{i-1}^{*}\right)$ and $\Delta=\max _{1 \leq i \leq n}\left|t_{i}^{*}-t_{i-1}^{*}\right|$. Then, the definite integral of $x(t)$ over $\left[t_{0}, t_{0}+a\right]$ is defined by $\int_{t_{0}}^{t_{0}+a} x(t) d t=\lim _{\Delta \rightarrow 0} \mathfrak{R}_{\wp}$ provided that the limit exists in the metric space $\left(\mathbb{R}_{\mathscr{F}}, d_{\infty}\right)$.

Theorem 8 (see [28]). Let $x:\left[t_{0}, t_{0}+a\right] \rightarrow \mathbb{R}_{\mathscr{F}}$ be continuous fuzzy-valued function and put $[x(t)]^{r}=\left[\underline{x}_{r}(t), \bar{x}_{r}(t)\right]$ for each $r \in[0,1]$. Then, $\int_{t_{0}}^{t_{0}+a} x(t) d t$ exist, belonging to $\mathbb{R}_{\mathscr{F}}, \underline{x}_{r}$ and $\bar{x}_{r}$ are integrable functions on $\left[t_{0}, t_{0}+a\right]$, and $\left[\int_{t_{0}}^{t_{0}+a} x(t) d t\right]^{r}=$ $\left[\int_{t_{0}}^{t_{0}+a} \underline{x}_{r}(t) d t, \int_{t_{0}}^{t_{0}+a} \bar{x}_{r}(t) d t\right]$

Lemma 9 (see $[31])$. Let $x, y:\left[t_{0}, t_{0}+a\right] \rightarrow \mathbb{R}_{\mathscr{F}}$ be integrable fuzzy-valued function and $\lambda \in \mathbb{R}$. Then, the following hold:

(i) $d_{\infty}(x(t), y(t))$ is integrable; 
(ii) $d_{\infty}\left(\int_{t_{0}}^{t_{0}+a} x(t) d t, \int_{t_{0}}^{t_{0}+a} y(t) d t\right) \leq \int_{t_{0}}^{t_{0}+a} d_{\infty}(x(t), y(t)) d t$;

(iii) $\int_{t_{0}}^{t_{0}+a} \lambda x(t) d t=\lambda \int_{t_{0}}^{t_{0}+a} x(t) d t$;

(iv) $\int_{t_{0}}^{t_{0}+a}(x(t)+y(t)) d t=\int_{t_{0}}^{t_{0}+a} x(t) d t+\int_{t_{0}}^{t_{0}+a} y(t) d t$.

It should be noted that the fuzzy integral can be also defined using the Lebesgue-type approach [27] or the Henstock-type approach [32]. However, if $x$ is continuous function, then all approaches yield the same value and results. Moreover, the representation of the fuzzy integral using Definition 7 is more convenient for numerical calculations and computational mathematics. The reader is kindly requested to go through $[27,28,31-33]$ in order to know more details about the fuzzy integrals, including their history and kinds, their properties and modification for use, their applications and characteristics, their justification and conditions for use, and their mathematical and geometric properties.

\section{Solving Fuzzy Volterra Integrodifferential Equation}

The topic of fuzzy VIDEs is one of the most important modern mathematical fields that result from modeling of uncertain physical, engineering, and economical problems. In this section, we study fuzzy VIDEs using the concept of strongly generalized differentiability in which fuzzy equation is converted into equivalent system of crisp equations for each type of differentiability. Furthermore, we present an algorithm to solve the new system which consists of two crisp VIDEs.

Let us consider the following first-order equation describing the crisp VIDE:

$$
x^{\prime}(t)=f(t, x(t))+\int_{t_{0}}^{t} g(t, \tau, x(\tau)) d \tau, \quad t_{0} \leq t, \tau \leq t_{0}+a,
$$

subject to the crisp initial condition,

$$
x\left(t_{0}\right)=\sigma
$$

where $f:\left[t_{0}, t_{0}+a\right] \times \mathbb{R} \rightarrow \mathbb{R}$ and $g:\left[t_{0}, t_{0}+a\right]^{2} \times \mathbb{R} \rightarrow \mathbb{R}$ are continuous real-valued function and $\sigma, t_{0}, a \in \mathbb{R}$ with $a>0$.

Assume that the initial condition $\sigma$ in (5) is uncertain and modeled by a fuzzy number. Also, assume that the functions $f$ and $g$ in (4) contain uncertain parameters modeled by a fuzzy number. Then, we obtain the fuzzy VIDEs (1) and (2). In order to solve this problem, we write the fuzzy function $x(t)$ in terms of its $r$-cut representation form to get $[x(t)]^{r}=$ $\left[\underline{x}_{r}(t), \bar{x}_{r}(t)\right]$ and $\left[x\left(t_{0}\right)\right]^{r}=\left[\underline{\sigma}_{r}, \bar{\sigma}_{r}\right]$.

On the other aspect as well, the Zadeh extension principle will lead to the following definition of $f(t, x(t))$ when $x(t)$ is a fuzzy number [34]: $f(t, x(t))(s)=\sup \{x(t)(\tau): s=$ $f(t, \tau), s \in \mathbb{R}\}$. Indeed, according to Nguyen theorem [35] it follows that

$$
\begin{aligned}
{[f(t, x(t))]^{r} } & =f\left(t,[x(t)]^{r}\right) \\
& =\left\{f(t, y): y \in[x(t)]^{r}\right\} \\
& =\left[\underline{f}_{r}(t, x(t)), \bar{f}_{r}(t, x(t))\right],
\end{aligned}
$$

where the two term endpoints functions $f_{r}$ and $\bar{f}_{r}$ are defined, respectively, as $\underline{f}_{r}(t, x(t)):=\min \{\hat{f}(t, y): y \in$ $\left.[x(t)]^{r}\right\}=f_{1, r}\left(t, \underline{x}_{r}(t), \bar{x}_{r}(t)\right)$ and $\bar{f}_{r}(t, x(t)):=\max \{f(t, y):$ $\left.y \in[x(t)]^{r}\right\}=\bar{f}_{2, r}\left(t, \underline{x}_{r}(t), \bar{x}_{r}(t)\right)$. Similarly, the parametric form of $g$ is given as

$$
\begin{aligned}
{[g(t, \tau, x(\tau))]^{r}=} & {\left[\underline{g}_{r}(t, \tau, x(t)), \bar{g}_{r}(t, \tau, x(t))\right] } \\
= & {\left[g_{1, r}\left(t, \tau, \underline{x}_{r}(\tau), \bar{x}_{r}(\tau)\right),\right.} \\
& \left.g_{2, r}\left(t, \tau, \underline{x}_{r}(\tau), \bar{x}_{r}(\tau)\right)\right] .
\end{aligned}
$$

The reader is asked to refer to [34-37] in order to know more details about Zadeh's extension principle and Nguyen theorem, including their justification and conditions for use, their mathematical and geometric properties, their types and kinds, and their applications and method of calculations.

Definition 10. Let $x:\left[t_{0}, t_{0}+a\right] \rightarrow \mathbb{R}_{\mathscr{F}}$ such that $D_{1} x$ or $D_{2} x$ exists. If $x$ and $D_{1} x$ satisfy fuzzy VIDEs (1) and (2), one says that $x$ is a (1)-solution of fuzzy VIDEs (1) and (2). Similarly, if $x$ and $D_{2} x$ satisfy fuzzy VIDEs (1) and (2), one says that $x$ is a (2)-solution of fuzzy VIDEs (1) and (2).

The object of the next algorithm is to implement a procedure to solve fuzzy VIDE in parametric form in terms of its $r$-cut representation, where the new obtained system consists of two crisp VIDEs for each type of differentiability.

Algorithm 11. To find solutions of fuzzy VIDEs (1) and (2), we discuss the following two cases.

Case 1. If $x(t)$ is (1)-differentiable, then $\left[D_{1} x(t)\right]^{r}=$ $\left[\underline{x}_{r}^{\prime}(t), \bar{x}_{r}^{\prime}(t)\right]$ and solving fuzzy VIDEs (1) and (2) translates into the following subroutine.

Step 1. Solve the following system of crisp VIDEs for $\underline{x}_{r}(t)$ and $\bar{x}_{r}(t)$ :

$$
\begin{aligned}
& \underline{x}_{r}^{\prime}(t)=f_{1, r}\left(t, \underline{x}_{r}(t), \bar{x}_{r}(t)\right)+\int_{t_{0}}^{t} g_{1, r}\left(t, \tau, \underline{x}_{r}(\tau), \bar{x}_{r}(\tau)\right) d \tau, \\
& \bar{x}_{r}^{\prime}(t)=f_{2, r}\left(t, \underline{x}_{r}(t), \bar{x}_{r}(t)\right)+\int_{t_{0}}^{t} g_{2, r}\left(t, \tau, \underline{x}_{r}(\tau), \bar{x}_{r}(\tau)\right) d \tau,
\end{aligned}
$$

subject to the crisp initial conditions,

$$
\underline{x}_{r}\left(t_{0}\right)=\underline{\sigma}_{r}, \quad \bar{x}_{r}\left(t_{0}\right)=\bar{\sigma}_{r} .
$$


Step 2. Ensure that the solution $\left[\underline{x}_{r}(t), \bar{x}_{r}(t)\right]$ and its derivative $\left[\underline{x}_{r}^{\prime}(t), \bar{x}_{r}^{\prime}(t)\right]$ are valid level sets for each $r \in[0,1]$.

Step 3. Use (3) to construct a (1)-solution $x(t)$ such that $[x(t)]^{r}=\left[\underline{x}_{r}(t), \bar{x}_{r}(t)\right]$ for each $r \in[0,1]$.

Case 2. If $x(t)$ is (2)-differentiable, then $\left[D_{2} x(t)\right]^{r}=$ $\left[\bar{x}_{r}^{\prime}(t), \underline{x}_{r}^{\prime}(t)\right]$ and solving fuzzy VIDEs (1) and (2) translates into the following subroutine.

Step 1. Solve the following system of crisp VIDEs for $\underline{x}_{r}(t)$ and $\bar{x}_{r}(t)$ :

$$
\begin{aligned}
& \underline{x}_{r}^{\prime}(t)=f_{2, r}\left(t, \underline{x}_{r}(t), \bar{x}_{r}(t)\right)+\int_{t_{0}}^{t} g_{2, r}\left(t, \tau, \underline{x}_{r}(\tau), \bar{x}_{r}(\tau)\right) d \tau, \\
& \bar{x}_{r}^{\prime}(t)=f_{1, r}\left(t, \underline{x}_{r}(t), \bar{x}_{r}(t)\right)+\int_{t_{0}}^{t} g_{1, r}\left(t, \tau, \underline{x}_{r}(\tau), \bar{x}_{r}(\tau)\right) d \tau,
\end{aligned}
$$

subject to the crisp initial conditions,

$$
\underline{x}_{r}\left(t_{0}\right)=\underline{\sigma}_{r}, \quad \bar{x}_{r}\left(t_{0}\right)=\bar{\sigma}_{r} .
$$

Step 2. Ensure that the solution $\left[\underline{x}_{r}(t), \bar{x}_{r}(t)\right]$ and its derivative $\left[\bar{x}_{r}^{\prime}(t), \underline{x}_{r}^{\prime}(t)\right]$ are valid level sets for each $r \in[0,1]$.

Step 3. Use (3) to construct a (2)-solution $x(t)$ such that $[x(t)]^{r}=\left[\underline{x}_{r}(t), \bar{x}_{r}(t)\right]$ for each $r \in[0,1]$.

Sometimes, we cannot decompose the membership function of the fuzzy solution $[x(t)]^{r}$ as a function defined on $\mathbb{R}$ for each $t \in\left[t_{0}, t_{0}+a\right]$. Then, using identity (3), we can leave the solution in terms of its $r$-cut representation form.

Next, we construct a procedure based on Algorithm 11 in order to obtain the analytic or the approximate solutions of fuzzy VIDEs (1) and (2). Anyhow, by considering the (1)differentiability concept and without loss of generality, we assume that the function $g$ takes the form $g(t, \tau, x(\tau))=$ $k(t, \tau) G(x(\tau))$. So, based on this, fuzzy VIDE (1) can be written in a new discretized form as $x^{\prime}(t)=f(t, x(t))+$ $\int_{t_{0}}^{t} k(t, \tau) G(x(\tau)) d \tau$, in which the $r$-cut representation form of $G(x(\tau))$ should be of the form

$$
\begin{aligned}
{[G(x(\tau))]^{r} } & =\left[\underline{G}_{r}(x(\tau)), \bar{G}_{r}(x(\tau))\right] \\
& =\left[G_{1, r}\left(\underline{x}_{r}(\tau), \bar{x}_{r}(\tau)\right), G_{2, r}\left(\underline{x}_{r}(\tau), \bar{x}_{r}(\tau)\right)\right] .
\end{aligned}
$$

In order to design a scheme for solving fuzzy VIDEs (1) and (2), we first replace it by the following equivalent crisp system of VIDEs:

$$
\begin{aligned}
& \underline{x}_{r}^{\prime}(t)=f_{1, r}\left(t, \underline{x}_{r}(t), \bar{x}_{r}(t)\right)+\int_{t_{0}}^{t} K_{1, r}\left(t, \tau, \underline{x}_{r}(\tau), \bar{x}_{r}(\tau)\right) d \tau, \\
& \underline{x}_{r}^{\prime}(t)=f_{1, r}\left(t, \underline{x}_{r}(t), \bar{x}_{r}(t)\right)+\int_{t_{0}}^{t} K_{2, r}\left(t, \tau, \underline{x}_{r}(\tau), \bar{x}_{r}(\tau)\right) d \tau,
\end{aligned}
$$

subject to the crisp initial conditions,

$$
\underline{x}_{r}\left(t_{0}\right)=\underline{\sigma}_{r}, \quad \bar{x}_{r}\left(t_{0}\right)=\bar{\sigma}_{r}
$$

where the new functions $K_{1, r}, K_{2, r}$ are given, respectively, as

$$
\begin{aligned}
K_{1, r}\left(t, \tau, \underline{x}_{r}(\tau), \bar{x}_{r}(\tau)\right) \\
\quad= \begin{cases}k(t, \tau) G_{1, r}\left(\underline{x}_{r}(\tau), \bar{x}_{r}(\tau)\right), & k(t, \tau) \geq 0, \\
k(t, \tau) G_{2, r}\left(\underline{x}_{r}(\tau), \bar{x}_{r}(\tau)\right), & k(t, \tau)<0,\end{cases} \\
K_{2, r}\left(t, \tau, \underline{x}_{r}(\tau), \bar{x}_{r}(\tau)\right) \\
= \begin{cases}k(t, \tau) G_{2, r}\left(\underline{x}_{r}(\tau), \bar{x}_{r}(\tau)\right), & k(t, \tau) \geq 0, \\
k(t, \tau) G_{1, r}\left(\underline{x}_{r}(\tau), \bar{x}_{r}(\tau)\right), & k(t, \tau)<0 .\end{cases}
\end{aligned}
$$

Prior to applying the analytic or the numerical methods for solving system of crisp VIDEs (13) and (14), we suppose that the kernel function $k(t, \tau)$ is nonnegative for $t_{0} \leq \tau \leq$ $c$ and nonpositive for $c \leq \tau \leq t$. Therefore, system of crisp VIDEs (13) can be translated again into the following equivalent form:

$$
\begin{aligned}
\underline{x}_{r}^{\prime}(t)= & f_{1, r}\left(t, \underline{x}_{r}(t), \bar{x}_{r}(t)\right) \\
& +\int_{t_{0}}^{c} k(t, \tau) G_{1, r}\left(\underline{x}_{r}(\tau), \bar{x}_{r}(\tau)\right) d \tau \\
& +\int_{c}^{t} k(t, \tau) G_{2, r}\left(\underline{x}_{r}(\tau), \bar{x}_{r}(\tau)\right) d \tau, \\
\bar{x}_{r}^{\prime}(t)= & f_{2, r}\left(t, \underline{x}_{r}(t), \bar{x}_{r}(t)\right) \\
& +\int_{t_{0}}^{c} k(t, \tau) G_{2, r}\left(\underline{x}_{r}(\tau), \bar{x}_{r}(\tau)\right) d \tau \\
& +\int_{c}^{t} k(t, \tau) G_{1, r}\left(\underline{x}_{r}(\tau), \bar{x}_{r}(\tau)\right) d \tau .
\end{aligned}
$$

Similarly, if $x$ is (2)-differentiable, then fuzzy VIDEs (1) can be translated into the form

$$
\begin{aligned}
\underline{x}_{r}^{\prime}(t)= & f_{2, r}\left(t, \underline{x}_{r}(t), \bar{x}_{r}(t)\right) \\
& +\int_{t_{0}}^{c} k(t, \tau) G_{2, r}\left(\underline{x}_{r}(\tau), \bar{x}_{r}(\tau)\right) d \tau \\
& +\int_{c}^{t} k(t, \tau) G_{1, r}\left(\underline{x}_{r}(\tau), \bar{x}_{r}(\tau)\right) d \tau, \\
\bar{x}_{r}^{\prime}(t)= & f_{1, r}\left(t, \underline{x}_{r}(t), \bar{x}_{r}(t)\right) \\
& +\int_{t_{0}}^{c} k(t, \tau) G_{1, r}\left(\underline{x}_{r}(\tau), \bar{x}_{r}(\tau)\right) d \tau \\
& +\int_{c}^{t} k(t, \tau) G_{2, r}\left(\underline{x}_{r}(\tau), \bar{x}_{r}(\tau)\right) d \tau,
\end{aligned}
$$

subject to the same crisp initial conditions of (14). 


\section{Existence and Uniqueness of Two Fuzzy Solutions}

The topics of fuzzy VIDEs which is growing interest for some time, in particular in relation to fuzzy control, fuzzy population growth model, and fuzzy oscillating magnetic fields, have been rapidly developed in recent years. Anyhow, in this work, we are interested in the following main questions; firstly, under what conditions can we be sure that solutions of fuzzy VIDEs (1) and (2) exist? Secondly, under what conditions can we be sure that there are two unique solutions, one solution for each lateral derivative, to fuzzy VIDEs (1) and (2)?

Remark 12. Throughout this paper, we will try to give the results of the all theorems and lemmas; however, in some cases, we will switch between the results obtained for the two types of differentiability in order not to increase the length of the paper without loss of generality for the remaining results. Actually, in the same manner, we can employ the same technique to construct the proof for the omitted case.

A fuzzy-valued function $f:\left[t_{0}, t_{0}+a\right] \times \mathbb{R}_{\mathscr{F}} \rightarrow \mathbb{R}_{\mathscr{F}}$ is called continuous at a point $\left(t^{*}, z^{*}\right)$ in $\left[t_{0}, t_{0}+a\right] \times \mathbb{R}_{\mathscr{F}}$ provided for arbitrary $\varepsilon>0$; there exists an $\delta(\varepsilon, r)>0$ such that $d_{\infty}\left(f(t, z), f\left(t^{*}, z^{*}\right)\right)<\varepsilon$ whenever $\left|t^{*}-t\right|<\delta$ and $d_{\infty}\left(z, z^{*}\right)<\delta$ for each $t \in\left[t_{0}, t_{0}+a\right]$ and $z \in \mathbb{R}_{\mathscr{F}}$. Similarly, for $g:\left[t_{0}, t_{0}+a\right]^{2} \times \mathbb{R}_{\mathscr{F}} \rightarrow \mathbb{R}_{\mathscr{F}}$ with the need for attention to change the metric use on $\left[t_{0}, t_{0}+a\right]^{2} \times \mathbb{R}_{\mathscr{F}}$.

Denote by $C\left(\left[t_{0}, t_{0}+a\right], \mathbb{R}_{\mathscr{F}}\right)$ the set of all continuous mapping from $\left[t_{0}, t_{0}+a\right]$ to $\mathbb{R}_{\mathscr{F}}$. The supremum metric on $\mathcal{C}\left(\left[t_{0}, t_{0}+a\right], \mathbb{R}_{\mathscr{F}}\right)$ is defined by $d_{1}: \mathcal{C}\left(\left[t_{0}, t_{0}+a\right], \mathbb{R}_{\mathscr{F}}\right) \times$ $\mathcal{C}\left(\left[t_{0}, t_{0}+a\right], \mathbb{R}_{\mathscr{F}}\right) \rightarrow \mathbb{R}^{+} \cup\{0\}$ such that $d_{1}(x, y)=$ $\sup _{t \in\left[t_{0}, t_{0}+a\right]}\left(d_{\infty}(x(t), y(t)) e^{-\eta t}\right)$ for each $x, y \in \mathcal{C}\left(\left[t_{0}, t_{0}+\right.\right.$ $\left.a], \mathbb{R}_{\mathscr{F}}\right)$, where $\eta \in \mathbb{R}$ is fixed. It is shown in [38] that $\left(\complement\left(\left[t_{0}, t_{0}+a\right], \mathbb{R}_{\mathscr{F}}\right), d_{1}\right)$ is a complete metric space.

The following lemma transforms a fuzzy VIDE into two fuzzy integral equations. Here, the equivalence between two equations means that any solution of an equation is a solution too for the other one with respect to the differentiability used.

Lemma 13. The fuzzy VIDEs (1) and (2), where $f:\left[t_{0}, t_{0}+a\right] \times$ $\mathbb{R}_{\mathscr{F}} \rightarrow \mathbb{R}_{\mathscr{F}}$ and $g:\left[t_{0}, t_{0}+a\right]^{2} \times \mathbb{R}_{\mathscr{F}} \rightarrow \mathbb{R}_{\mathscr{F}}$ are supposed to be continuous, are equivalent to one of the following fuzzy integral equations:

(i) $x(t)=\sigma+\int_{t_{0}}^{t} f(s, x(s)) d s+\int_{t_{0}}^{t}\left(\int_{t_{0}}^{s} g(s, \tau, x(\tau)) d \tau\right) d s$;

(ii) $x(t)=\sigma \ominus(-1) \int_{t_{0}}^{t} f(s, x(s)) d s \ominus(-1) \int_{t_{0}}^{t}\left(\int_{t_{0}}^{s} g(s, \tau\right.$, $x(\tau)) d \tau) d s$,

depending on the strong differentiability considered, (1)differentiability or (2)-differentiability, respectively.

Proof. Since $f$ and $g$ are continuous functions, so they are integrable. Now, we determine the equivalent integral forms of fuzzy VIDEs (1) and (2) under each type of strongly generalized differentiability as follows. Firstly, let us consider that $x$ is (1)-differentiable; then the equivalent integral form of fuzzy VIDEs (1) and (2) can be written by implementation of fuzzy integration on both sides of the original equation one time as follows:

$$
\begin{aligned}
x(t)= & x\left(t_{0}\right)+\int_{t_{0}}^{t} f(s, x(s)) d s \\
& +\int_{t_{0}}^{t}\left(\int_{t_{0}}^{s} g(s, \tau, x(\tau)) d \tau\right) d s .
\end{aligned}
$$

Secondly, let us consider that $x$ is (2)-differentiable; then the equivalent integral form of fuzzy VIDEs (1) and (2) can be written as

$$
\begin{aligned}
x\left(t_{0}\right)= & x(t)+(-1) \int_{t_{0}}^{t} f(s, x(s)) d s \\
& +(-1) \int_{t_{0}}^{t}\left(\int_{t_{0}}^{s} g(s, \tau, x(\tau)) d \tau\right) d s
\end{aligned}
$$

which is equivalent to the form of part (ii) of Lemma 13.

Lemma 13 can be reformulated as follows: a mapping $x$ : $\left[t_{0}, t_{0}+a\right] \rightarrow \mathbb{R}_{\mathscr{F}}$ is a solution to the fuzzy VIDEs (1) and (2) if and only if $x$ is continuous and satisfy Case (i) or Case (ii) in the sense of (1)-differentiability or in the sense of (2)differentiability, respectively.

In mathematics, the Banach fixed-point theorem, also known as the contraction mapping theorem, is an important tool in the theory of metric spaces; it guarantees the existence and uniqueness of fixed points of certain self-maps of metric spaces and provides a constructive method to find those fixed points. The following results (Definition 14 and Theorem 15) were collected from [39].

Definition 14. Let $(X, d)$ be a metric space. A mapping $G$ : $X \rightarrow X$ is said to be a contraction mapping, if there exists a positive real number $\alpha$ with $\alpha<1$ such that $d(G(x), G(y)) \leq$ $\alpha d(x, y)$ for each $x, y \in X$.

We observe that applying $G$ to each of the two points of the space contracts the distance between them; obviously, $G$ is continuous. Anyhow, a point $x \in X$ is called a fixed point of the mapping $G: X \rightarrow X$ if $G(x)=x$. Next, we present the Banach fixed-point theorem.

Theorem 15. Any contraction mapping $G$ of a nonempty complete metric space $(X, d)$ into itself has a unique fixed point.

Lemma 16. The real-valued functions $v, \omega:\left[t_{0}, t_{0}+a\right] \rightarrow \mathbb{R}$ with $\eta \in \mathbb{R}$, represented by

$$
\begin{aligned}
& \nu(t)=\frac{1}{\eta^{2}}\left(1-e^{-\eta\left(t-t_{0}\right)}-\eta\left(t-t_{0}\right) e^{-\eta\left(t-t_{0}\right)}\right), \\
& \omega(t)=\frac{1}{\eta}\left(1-e^{-\eta\left(t-t_{0}\right)}\right),
\end{aligned}
$$

are continuous nondecreasing functions on $\left[t_{0}, t_{0}+a\right]$. Furthermore, $v\left(t_{0}+a\right)=\sup _{t \in\left[t_{0}, t_{0}+a\right]} v(t), \omega\left(t_{0}+a\right)=\sup _{t \in\left[t_{0}, t_{0}+a\right]} \omega(t)$, and $\lim _{\eta \rightarrow+\infty}\left(\nu\left(t_{0}+a\right)+\omega\left(t_{0}+a\right)\right)=0$. 
Proof. Clearly $v, \omega$ are continuous functions on $\left[t_{0}, t_{0}+a\right]$ for each $\eta \in \mathbb{R}$. Since $\nu^{\prime}(t)=\left(t-t_{0}\right) e^{-\eta\left(t-t_{0}\right)}>0$ and $\omega^{\prime}(t)=e^{-\eta\left(t-t_{0}\right)}>0$ for each $t \in\left[t_{0}, t_{0}+a\right]$ and $\eta \in \mathbb{R}$, thus, $v, \omega$ are nondecreasing functions. As a result, one can conclude that $\nu\left(t_{0}+a\right)=\sup _{t \in\left[t_{0}, t_{0}+a\right]} \nu(t)$ and $\omega\left(t_{0}+a\right)=$ $\sup _{t \in\left[t_{0}, t_{0}+a\right]} \omega(t)$. On the other aspect as well, using the limit functions techniques, yields that

$$
\begin{aligned}
\lim _{\eta \rightarrow+\infty} & \left(\nu\left(t_{0}+a\right)+\omega\left(t_{0}+a\right)\right) \\
= & \lim _{\eta \rightarrow+\infty}\left(\frac{1}{\eta^{2}}\left(1-e^{-\eta a}-\eta a e^{-\eta a}\right)+\frac{1}{\eta}\left(1-e^{-\eta a}\right)\right) \\
= & \lim _{\eta \rightarrow+\infty} \frac{1}{\eta}\left(\frac{1}{\eta}+1-\frac{1}{\eta} e^{-\eta a}-e^{-\eta a}-a e^{-\eta a}\right)=0 .
\end{aligned}
$$

It should be mentioned here that Lemma 16 guarantees the existence of a unique fixed point for the next theorem. In other words, an existence of a unique solution for fuzzy VIDEs (1) and (2) for each type of differentiability.

Theorem 17. Let $f:\left[t_{0}, t_{0}+a\right] \times \mathbb{R}_{\mathscr{F}} \rightarrow \mathbb{R}_{\mathscr{F}}$ and $g:\left[t_{0}, t_{0}+\right.$ $a]^{2} \times \mathbb{R}_{\mathscr{F}} \rightarrow \mathbb{R}_{\mathscr{F}}$ be continuous fuzzy-valued functions. If there exists $K_{1}, K_{2}>0$ such that

$$
\begin{gathered}
d_{\infty}\left(f\left(t, \xi_{1}(t)\right), f\left(t, \xi_{2}(t)\right)\right) \leq K_{1} d_{\infty}\left(\xi_{1}(t), \xi_{2}(t)\right), \\
d_{\infty}\left(g\left(t, \tau, \xi_{1}(\tau)\right), g\left(t, \tau, \xi_{2}(\tau)\right)\right) \leq K_{2} d_{\infty}\left(\xi_{1}(\tau), \xi_{2}(\tau)\right),
\end{gathered}
$$

for each $t, \tau \in\left[t_{0}, t_{0}+a\right], r \in[0,1]$, and $\xi_{1,2}(t), \xi_{1,2}(\tau) \in \mathbb{R}_{\mathscr{F}}$, then, the fuzzy VIDEs (1) and (2) have two unique solutions on $\left[t_{0}, t_{0}+a\right]$. One is (1)-differentiable solution and the other one is (2)-differentiable solution.

Proof. Without loss of generality, we consider the (1)differentiability only; actually, in the same manner, we can employ the same technique for the (2)-differentiability. For each $\xi(t) \in \mathbb{R}_{\mathscr{F}}$ and $t \in\left[t_{0}, t_{0}+a\right]$, define the operator $G \xi$ as

$$
\begin{aligned}
(G \xi)(t)= & \sigma+\int_{t_{0}}^{t} f(s, \xi(s)) d s \\
& +\int_{t_{0}}^{t}\left(\int_{t_{0}}^{s} g(s, \tau, \xi(\tau)) d \tau\right) d s .
\end{aligned}
$$

Thus, $G \xi:\left[t_{0}, t_{0}+a\right] \rightarrow \mathbb{R}_{\mathscr{F}}$ is continuous and $G: C\left(\left[t_{0}, t_{0}+\right.\right.$ $\left.a], \mathbb{R}_{\mathscr{F}}\right) \rightarrow \complement\left(\left[t_{0}, t_{0}+a\right], \mathbb{R}_{\mathscr{F}}\right)$. Now, we are going to show that the operator $G \xi$ satisfies the hypothesis of the Banachfixed point theorem. For each $\xi_{1}, \xi_{2} \in \mathcal{C}\left(\left[t_{0}, t_{0}+a\right], \mathbb{R}_{\mathscr{F}}\right)$, we have

$$
\begin{aligned}
& d_{1}\left(G \xi_{1}, G \xi_{2}\right) \\
& =\sup _{t \in\left[t_{0}, t_{0}+a\right]}\left(d_{\infty}\left(\left(G \xi_{1}\right)(t),\left(G \xi_{2}\right)(t)\right) e^{-\eta t}\right)
\end{aligned}
$$

$$
\begin{aligned}
&=\sup _{t \in\left[t_{0}, t_{0}+a\right]}\left\{d_{\infty}(\sigma\right.+\int_{t_{0}}^{t} f\left(s, \xi_{1}(s)\right) d s \\
&+\int_{t_{0}}^{t}\left(\int_{t_{0}}^{s} g\left(s, \tau, \xi_{1}(\tau)\right) d \tau\right) d s, \\
&+\int_{t_{0}}^{t} f\left(s, \xi_{2}(s)\right) d s \\
&\left.\left.+\int_{t_{0}}^{t}\left(\int_{t_{0}}^{s} g\left(s, \tau, \xi_{2}(\tau)\right) d \tau\right) d s\right) e^{-\eta t}\right\} \\
&=\sup _{t \in\left[t_{0}, t_{0}+a\right]}\left\{d _ { \infty } \left(\int_{t_{0}}^{t} f\left(s, \xi_{1}(s)\right) d s\right.\right. \\
&+\int_{t_{0}}^{t}\left(\int_{t_{0}}^{s} g\left(s, \tau, \xi_{1}(\tau)\right) d \tau\right) d s, \\
& \int_{t_{0}}^{t} f\left(s, \xi_{2}(s)\right) d s \\
&\left.\left.+\int_{t_{0}}^{t}\left(\int_{t_{0}}^{s} g\left(s, \tau, \xi_{2}(\tau)\right) d \tau\right) d s\right) e^{-\eta t}\right\}
\end{aligned}
$$$$
\begin{aligned}
\leq \sup _{t \in\left[t_{0}, t_{0}+a\right]}\{ & d_{\infty}\left(\int_{t_{0}}^{t} f\left(s, \xi_{1}(s)\right) d s, \int_{t_{0}}^{t} f\left(s, \xi_{2}(s)\right) d s\right) e^{-\eta t} \\
+ & d_{\infty}\left(\int_{t_{0}}^{t}\left(\int_{t_{0}}^{s} g\left(s, \tau, \xi_{1}(\tau)\right) d \tau\right) d s\right. \\
& \left.\left.\int_{t_{0}}^{t}\left(\int_{t_{0}}^{s} g\left(s, \tau, \xi_{2}(\tau)\right) d \tau\right) d s\right) e^{-\eta t}\right\}
\end{aligned}
$$$$
\leq \sup _{t \in\left[t_{0}, t_{0}+a\right]}\left\{\int_{t_{0}}^{t} d_{\infty}\left(f\left(s, \xi_{1}(s)\right), f\left(s, \xi_{2}(s)\right)\right) d s e^{-\eta t}\right.
$$$$
+\int_{t_{0}}^{t} \int_{t_{0}}^{s} d_{\infty}\left(g\left(s, \tau, \xi_{1}(\tau)\right),\right.
$$$$
\left.\left.g\left(s, \tau, \xi_{2}(\tau)\right)\right) d \tau d s e^{-\eta t}\right\}
$$

$$
\begin{gathered}
\leq \sup _{t \in\left[t_{0}, t_{0}+a\right]}\left\{\int_{t_{0}}^{t} K_{1} d_{\infty}\left(\xi_{1}(s), \xi_{2}(s)\right) d s e^{-\eta t}\right. \\
\left.\quad+\int_{t_{0}}^{t} \int_{t_{0}}^{s} K_{2} d_{\infty}\left(\xi_{1}(\tau), \xi_{2}(\tau)\right) d \tau d s e^{-\eta t}\right\} \\
\leq \sup _{t \in\left[t_{0}, t_{0}+a\right]}\left\{\int_{t_{0}}^{t} K_{1} d_{1}\left(\xi_{1}, \xi_{2}\right) e^{s t} d s e^{-\eta t}\right. \\
\left.+\int_{t_{0}}^{t} \int_{t_{0}}^{s} K_{2} d_{1}\left(\xi_{1}, \xi_{2}\right) e^{s \tau} d \tau d s e^{-\eta t}\right\}
\end{gathered}
$$

$\leq d_{1}\left(\xi_{1}, \xi_{2}\right)$

$$
\times \sup _{t \in\left[t_{0}, t_{0}+a\right]}\left\{\int_{t_{0}}^{t} K_{1} e^{s t} d s e^{-\eta t}+\int_{t_{0}}^{t} \int_{t_{0}}^{s} K_{2} e^{s \tau} d \tau d s e^{-\eta t}\right\}
$$

$\leq \max \left\{K_{1}, K_{2}\right\} d_{1}\left(\xi_{1}, \xi_{2}\right)$ 


$$
\begin{aligned}
& \times \sup _{t \in\left[t_{0}, t_{0}+a\right]}\left\{e^{-\eta t} \int_{t_{0}}^{t} e^{\eta s} d s+e^{-\eta t} \int_{t_{0}}^{t} \int_{t_{0}}^{s} e^{\eta \tau} d \tau d s\right\} \\
& =\max \left\{K_{1}, K_{2}\right\} d_{1}\left(\xi_{1}, \xi_{2}\right) \\
& \times \sup _{t \in\left[t_{0}, t_{0}+a\right]}\left\{e^{-\eta t}\left(\frac{1}{\eta^{2}}\left(e^{\eta t}-e^{\eta t_{0}}-\eta\left(t-t_{0}\right) e^{\eta t_{0}}\right)\right)\right. \\
& \left.+e^{-\eta t}\left(\frac{1}{\eta}\left(e^{\eta t}-e^{\eta t_{0}}\right)\right)\right\} \\
& =\max \left\{K_{1}, K_{2}\right\} d_{1}\left(\xi_{1}, \xi_{2}\right) \\
& \times \sup _{t \in\left[t_{0}, t_{0}+a\right]}\left\{\left(\frac{1}{\eta^{2}}\left(1-e^{-\eta\left(t-t_{0}\right)}-\eta\left(t-t_{0}\right) e^{-\eta\left(t-t_{0}\right)}\right)\right)\right. \\
& \left.+\left(\frac{1}{\eta}\left(1-e^{-\eta\left(t-t_{0}\right)}\right)\right)\right\} \\
& =\max \left\{K_{1}, K_{2}\right\} d_{1}\left(\xi_{1}, \xi_{2}\right)\left\{\left(\frac{1}{\eta^{2}}\left(1-e^{-\eta a}-\eta\left(t-t_{0}\right) e^{-\eta a}\right)\right)\right. \\
& \left.+\left(\frac{1}{\eta}\left(1-e^{-\eta a}\right)\right)\right\} \\
& =\max \left\{K_{1}, K_{2}\right\} \frac{1}{\eta}\left(\frac{1}{\eta}+1-\frac{1}{\eta} e^{-\eta a}-e^{-\eta a}-a e^{-\eta a}\right) \\
& \times d_{1}\left(\xi_{1}, \xi_{2}\right) \text {. }
\end{aligned}
$$

But since $\lim _{\eta \rightarrow+\infty}(1 / \eta)\left((1 / \eta)+1-(1 / \eta) e^{-\eta a}-e^{-\eta a}-a e^{-\eta a}\right)=$ 0 from Lemma 16, so, we can choose $\eta>0$ such that

$$
\max \left\{K_{1}, K_{2}\right\} \frac{1}{\eta}\left(\frac{1}{\eta}+1-\frac{1}{\eta} e^{-\eta a}-e^{-\eta a}-a e^{-\eta a}\right)<1 .
$$

Anyhow, $G$ is a contractive mapping, whilst the unique fixed point of $G$ is in the space $\complement\left(\left[t_{0}, t_{0}+a\right], \mathbb{R}_{\mathscr{F}}\right)$. Using the fact that $G \xi$ is the integral of a continuous function, we conclude that it is actually in the space $\mathcal{C}\left(\left[t_{0}, t_{0}+a\right], \mathbb{R}_{\mathscr{F}}\right)$. Hence, by the Banach fixed-point theorem, fuzzy VIDEs (1) and (2) have a unique fixed point $x \in \mathcal{C}\left(\left[t_{0}, t_{0}+a\right], \mathbb{R}_{\mathscr{F}}\right)$, that is, a continuous function $x$ on $\left[t_{0}, t_{0}+a\right]$ satisfying $G x=x$. As a result, writing $(G x)(t)=x(t)$ out, we have by $(23)$

$$
x(t)=\sigma+\int_{t_{0}}^{t} f(s, x(s)) d s+\int_{t_{0}}^{t}\left(\int_{t_{0}}^{s} g(s, \tau, x(\tau)) d \tau\right) d s .
$$

On the other aspect as well, differentiate both sides of (26) and substitute $t=t_{0}$ to obtain fuzzy VIDEs (1) and (2). Hence, every solution of fuzzy VIDEs (1) and (2) must satisfy (26), and conversely. So, the proof of the theorem is complete.

Remark 18. The continuous nonlinear terms $f:\left[t_{0}, t_{0}+a\right] \times$ $\mathbb{R}_{\mathscr{F}} \rightarrow \mathbb{R}_{\mathscr{F}}$ and $g:\left[t_{0}, t_{0}+a\right]^{2} \times \mathbb{R}_{\mathscr{F}} \rightarrow \mathbb{R}_{\mathscr{F}}$ are said to satisfy a Lipchitz condition relative to their last argument in fuzzy sense with respect to the metric space $\left(\mathbb{R}_{\mathscr{F}}, d_{\infty}\right)$ if the conditions of (22) of Theorem 17 hold.

\section{Generalized Characterization Theorem}

The characterization theorem shows us the following general hint on how to deal with the analytical or the numerical solutions of fuzzy VIDEs. We can translate the original fuzzy VIDE equivalently into a system of crisp VIDEs. The solutions techniques of the system of crisp VIDEs are extremely well studied in the literature, so any method we can consider for the system of crisp VIDEs, since the solution will be as well solution of the fuzzy VIDE under study. As a conclusion, one does not need to rewrite the methods for system of crisp VIDEs in fuzzy setting, but instead, we can use the methods directly on the obtained crisp system.

A function $f:\left[t_{0}, t_{0}+a\right] \times \mathbb{R}^{2} \rightarrow \mathbb{R}$ is said to be equicontinuous if, for any $\epsilon>0$ and any $(t, x, y) \in\left[t_{0}, t_{0}+\right.$ a] $\times \mathbb{R}^{2}$, we have $\left|f(t, x, y)-f\left(t, x_{1}, y_{1}\right)\right|<\epsilon$, whenever $\left\|\left(t, x_{1}, y_{1}\right)-(t, x, y)\right\|<\delta$, and uniformly bounded on any bounded set. Similarly, for a function defined on $\left[t_{0}, t_{0}+a\right]^{2} \times$ $\mathbb{R}^{2}$ with the need for attention to change the metric used on $\left[t_{0}, t_{0}+a\right]^{2} \times \mathbb{R}^{2}$.

Theorem 19. Consider the fuzzy VIDEs (1) and (2) where $f$ : $\left[t_{0}, t_{0}+a\right] \times \mathbb{R}_{\mathscr{F}} \rightarrow \mathbb{R}_{\mathscr{F}}$ and $g:\left[t_{0}, t_{0}+a\right]^{2} \times \mathbb{R}_{\mathscr{F}} \rightarrow \mathbb{R}_{\mathscr{F}}$ are such that

(i) $[f(t, x(t))]^{r}=\left[f_{1, r}\left(t, \underline{x}_{r}(t), \bar{x}_{r}(t)\right), f_{2, r}\left(t, \underline{x}_{r}(t), \bar{x}_{r}(t)\right)\right]$, and $[g(t, \tau, x(\tau))]^{r}=\left[g_{1, r}\left(t, \tau, \underline{x}_{r}(\tau), \bar{x}_{r}(\tau)\right), g_{2, r}(t, \tau\right.$, $\left.\left.\underline{x}_{r}(\tau), \bar{x}_{r}(\tau)\right)\right]$

(ii) $f_{1, r}, f_{2, r}$ and $g_{1, r}, g_{2, r}$ are equicontinuous functions and uniformly bounded on any bounded set;

(iii) there exists real-finite constants $L_{1}, L_{2}>0$ such that

$$
\begin{aligned}
& \left|f_{1,2, r}\left(t, \underline{x}_{r}(t), \bar{x}_{r}(t)\right)-f_{1,2, r}\left(t, \underline{y}_{r}(t), \bar{y}_{r}(t)\right)\right| \\
& \leq L_{1} \max \left\{\left|\underline{x}_{r}(t)-\underline{y}_{r}(t)\right|,\left|\bar{x}_{r}(t)-\bar{y}_{r}(t)\right|\right\}, \\
& \left|g_{1,2, r}\left(t, \tau, \underline{x}_{r}(\tau), \bar{x}_{r}(\tau)\right)-g_{1,2, r}\left(t, \tau, \underline{y}_{r}(\tau), \bar{y}_{r}(\tau)\right)\right| \\
& \leq L_{2} \max \left\{\left|\underline{x}_{r}(\tau)-\underline{y}_{r}(\tau)\right|,\left|\bar{x}_{r}(\tau)-\bar{y}_{r}(\tau)\right|\right\},
\end{aligned}
$$

for each $t, \tau \in\left[t_{0}, t_{0}+a\right], r \in[0,1]$, and $x(t), y(t), x(\tau), y(\tau) \in$ $\mathbb{R}_{\mathscr{F}}$. Then, for (1)-differentiability, the fuzzy VIDEs (1) and (2) and the system of crisp VIDEs (8) and (9) are equivalent; and, in (2)-differentiability, the fuzzy VIDEs (1) and (2) and the system of crisp VIDEs (10) and (11) are equivalent.

Proof. Since the proof procedure is similar for the two types of differentiability, assume that $x$ is (1)-differentiable without loss of generality. The equicontinuity of $f_{1, r}, f_{2, r}$ and $g_{1, r}, g_{2, r}$ implies the continuity of $f$ and $g$, respectively. Furthermore, the Lipchitz property of condition (iii) ensures that $f$ and $g$ satisfy a Lipchitz property in the metric space $\left(\mathbb{R}_{\mathscr{F}}, d_{\infty}\right)$ as follows:

$$
\begin{aligned}
d_{\infty}(f(t, x(t)), f(t, y(t))) \\
=\sup _{r \in[0,1]} d_{H}\left([f(t, x(t))]^{r},[f(t, y(t))]^{r}\right)
\end{aligned}
$$




$$
\begin{aligned}
& =\sup _{r \in[0,1]} \max \left\{\left|\underline{f}_{r}(t, x(t))-\underline{f}_{r}(t, y(t))\right|,\right. \\
& \left.\qquad\left|\bar{f}_{r}(t, x(t))-\bar{f}_{r}(t, y(t))\right|\right\} \\
& =\sup _{r \in[0,1]} \max \left\{\mid f_{1, r}\left(t, \underline{x}_{r}(t), \bar{x}_{r}(t)\right)\right. \\
& -\quad-f_{1, r}\left(t, \underline{y}_{r}(t), \bar{y}_{r}(t)\right) \mid, \\
& \qquad \mid f_{2, r}\left(t, \underline{x}_{r}(t), \bar{x}_{r}(t)\right) \\
& \left.\quad-f_{2, r}\left(t, \underline{y}_{r}(t), \bar{y}_{r}(t)\right) \mid\right\} \\
& \leq L_{1} \sup _{r \in[0,1]} \max \left\{\left|\underline{x}_{r}(t)-\underline{y}_{r}(t)\right|,\left|\bar{x}_{r}(t)-\bar{y}_{r}(t)\right|\right\} \\
& =L_{1} \sup _{r \in[0,1]} d_{H}\left([x(t)]^{r},[y(t)]^{r}\right) \\
& =L_{1} d_{\infty}(x(t), y(t)),
\end{aligned}
$$

while, on the other hand, by similar fashion, it is easy to conclude that

$$
d_{\infty}(g(t, \tau, x(\tau)), g(t, \tau, y(\tau))) \leq L_{2} d_{\infty}(x(\tau), y(\tau)) .
$$

By the continuity of $f$ and $g$, from the last Lipchitz conditions of (28) and (29), and the boundedness property of condition (ii), it follows that fuzzy VIDEs (1) and (2) have a unique solution on $\left[t_{0}, t_{0}+a\right]$. Whilst the solution of fuzzy VIDEs (1) and (2) is (1)-differentiable and so, by Theorem 6 Case (i), the functions $\underline{x}_{r}$ and $\bar{x}_{r}$ are differentiable on $\left[t_{0}, t_{0}+a\right]$, as a conclusion, one can obtained that $\left(\underline{x}_{r}(t), \bar{x}_{r}(t)\right)$ is a solution of crisp VIDEs (8) and (9).

Conversely, suppose that we have a solution $\left(\underline{x}_{r}(t), \bar{x}_{r}(t)\right)$ with $r \in[0,1]$ being fixed of fuzzy VIDEs (1) and (2) (note that this solution exists by property of condition (iii)), whilst the Lipchitz conditions of (28) and (29) imply the existence and uniqueness of fuzzy solution $\tilde{x}(t)$. Indeed, since $\tilde{x}$ is (1)differentiable, then $\underline{\tilde{x}}_{r}(t)$ and $\overline{\tilde{x}}_{r}(t)$ the endpoints of $[\tilde{x}(t)]^{r}$ are a solution of crisp VIDEs (8) and (9) (note that $[\tilde{x}]^{r}$ is obviously valid level sets of a fuzzy-valued function). But since the solution of crisp VIDEs (8) and (9) is unique, we have $[\tilde{x}(t)]^{r}=\left[\underline{\tilde{x}}_{r}(t), \overline{\tilde{x}}_{r}(t)\right]^{r}=\left[\underline{x}_{r}(t), \bar{x}_{r}(t)\right]^{r}=[x(t)]^{r}$. That is, the fuzzy VIDEs (1) and (2) and the system of crisp VIDEs (8) and (9) are equivalent. This completes the proof of the theorem.

The purpose of the next corollary is not to make an essential improvement of Theorem 19 but rather to give alternate conditions under which fuzzy VIDEs (1) and (2) and the corresponding system of crisp VIDEs are equivalent.

Corollary 20. Suppose that $f:\left[t_{0}, t_{0}+a\right] \times \mathbb{R}_{\mathscr{F}} \rightarrow \mathbb{R}_{\mathscr{F}}$ and $g:\left[t_{0}, t_{0}+a\right]^{2} \times \mathbb{R}_{\mathscr{F}} \rightarrow \mathbb{R}_{\mathscr{F}}$ are such that the condition (i) of
Theorem 19 holds. If there exists real-finite constants $L_{1}, L_{2}>0$ such that

$$
\begin{aligned}
& \left|f_{1,2, r}\left(t_{1}, \underline{x}_{r}\left(t_{1}\right), \bar{x}_{r}\left(t_{1}\right)\right)-f_{1,2, r}\left(t_{2}, \underline{y}_{r}\left(t_{2}\right), \bar{y}_{r}\left(t_{2}\right)\right)\right| \\
& \leq L_{1} \max \left\{\left|t_{2}-t_{1}\right|,\left|\underline{x}_{r}\left(t_{1}\right)-\underline{y}_{r}\left(t_{2}\right)\right|,\right. \\
& \left.\qquad \bar{x}_{r}\left(t_{1}\right)-\bar{y}_{r}\left(t_{2}\right) \mid\right\}, \\
& \left|g_{1,2, r}\left(t_{1}, \tau_{1}, \underline{x}_{r}\left(\tau_{1}\right), \bar{x}_{r}\left(\tau_{1}\right)\right)-g_{1,2, r}\left(t_{2}, \tau_{2}, \underline{y}_{r}\left(\tau_{2}\right), \bar{y}_{r}\left(\tau_{2}\right)\right)\right| \\
& \leq L_{2} \max \left\{\left|t_{1}-t_{2}\right|,\left|\tau_{1}-\tau_{2}\right|,\left|\underline{x}_{r}\left(\tau_{1}\right)-\underline{y}_{r}\left(\tau_{2}\right)\right|,\right. \\
& \left.\left|\bar{x}_{r}\left(\tau_{1}\right)-\bar{y}_{r}\left(\tau_{2}\right)\right|\right\},
\end{aligned}
$$

for each $t_{1,2}, \tau_{1,2} \in\left[t_{0}, t_{0}+a\right], r \in[0,1]$, and $x(t), y(t) \in \mathbb{R}_{\mathscr{F}}$, then, for (1)-differentiability, the fuzzy VIDEs (1) and (2) and the system of crisp VIDEs (8) and (9) are equivalent; and, in (2)-differentiability, the fuzzy VIDEs (1) and (2) and the system of crisp VIDEs (10) and (11) are equivalent.

Proof. Here, we consider the (1)-differentiability only; actually, in the same manner, we can employ the same technique in the sense of (2)-differentiability. To this end, assume the hypothesis of Corollary 20; then the conditions (i) and (iii) of Theorem 19 clearly hold. To establish condition (ii), apply the following: fix $\epsilon>0$, choose $\delta=\epsilon / L$, and suppose $\left\|\left(t, \underline{x}_{r}(t), \bar{x}_{r}(t)\right)-\left(t_{1}, \underline{y}_{r}\left(t_{1}\right), \bar{y}_{r}\left(t_{1}\right)\right)\right\|<\delta$. Then, for each $r \in[0,1]$, one can write

$$
\begin{aligned}
& \left|f_{1,2, r}\left(t, \underline{x}_{r}(t), \bar{x}_{r}(t)\right)-f_{1,2, r}\left(t_{1}, \underline{y}_{r}\left(t_{1}\right), \bar{y}_{r}\left(t_{1}\right)\right)\right| \\
& \quad \leq L_{1} \max \left\{\left|t-t_{1}\right|,\left|\underline{x}_{r}(t)-\underline{y}_{r}\left(t_{1}\right)\right|,\left|\bar{x}_{r}(t)-\bar{y}_{r}\left(t_{1}\right)\right|\right\} \\
& \quad \leq L_{1}\left\|\left(t, \underline{x}_{r}(t), \bar{x}_{r}(t)\right)-\left(t_{1}, \underline{y}_{r}\left(t_{1}\right), \bar{y}_{r}\left(t_{1}\right)\right)\right\| \\
& \quad \leq L_{1} \delta=\epsilon .
\end{aligned}
$$

Next, we want to show that $f_{1, r}, f_{2, r}$ are uniformly bounded on any bounded set. To do so, let $S$ be any bounded subset of $\left[t_{0}, t_{0}+a\right] \times \mathbb{R}^{2}$. Then, there exist constants $x_{1}, y_{1}, x_{2}, y_{2} \in \mathbb{R}$ such that if $w=(t, x(t), y(t)) \in S$, then $t \in\left[t_{0}, t_{0}+a\right], x(t) \in$ $\left[x_{1}, x_{2}\right]$ and $y(t) \in\left[y_{1}, y_{2}\right]$. For the conduct of proceedings in the proof, fix $r^{*} \in[0,1]$ and $w^{*} \in S$ and let $K=\max \left\{\mid t_{0}+\right.$ $\left.a-t_{0}|,| x_{2}-x_{1}|,| y_{2}-y_{1} \mid\right\}$ and $C=L_{1} K+\operatorname{supp} f\left(w^{*}\right)$, where supp $f\left(w^{*}\right)$ is the support of $f\left(w^{*}\right)$. Suppose that $r \in[0,1]$ and $w \in S$. Then, one can write

$$
\begin{aligned}
& \left|f_{1, r}(w)-f_{1, r}\left(w^{*}\right)\right| \\
& \quad \leq L_{1} \max \left\{\left|t_{0}+a-t_{0}\right|,\left|x_{2}-x_{1}\right|,\left|y_{2}-y_{1}\right|\right\}=L_{1} K
\end{aligned}
$$

while, on the other aspect as well, the triangle inequality will give

$$
\begin{aligned}
& \left|f_{1, r}(w)-f_{1, r^{*}}\left(w^{*}\right)\right| \\
& \quad=\left|f_{1, r}(w)-f_{1, r}\left(w^{*}\right)+f_{1, r}\left(w^{*}\right)-f_{1, r^{*}}\left(w^{*}\right)\right|
\end{aligned}
$$




$$
\begin{aligned}
& \leq\left|f_{1, r}(w)-f_{1, r}\left(w^{*}\right)\right|+\left|f_{1, r}\left(w^{*}\right)-f_{1, r^{*}}\left(w^{*}\right)\right| \\
& =L_{1} K+\operatorname{supp} f\left(w^{*}\right)=C .
\end{aligned}
$$

But since $\left|f_{1, r}(w)\right|-\left|f_{1, r^{*}}\left(w^{*}\right)\right| \leq\left|f_{1, r}(w)-f_{1, r^{*}}\left(w^{*}\right)\right| \leq C$ or $\left|f_{1, r}(w)\right| \leq C+\left|f_{1, r^{*}}\left(w^{*}\right)\right|$, therefore $f_{1, r}$ is uniformly bounded on $S$. Similarly, $f_{2, r}$ is uniformly bounded on any bounded set. The same procedure can be applied directly for $g_{1, r}, g_{2, r}$. Hence, fuzzy VIDEs (1) and (2) and system of crisp VIDEs (8) and (9) are equivalent by Theorem 19.

Remark 21. The readers should pay attention to the fact that the following requirement conditions on $f$ and $g$ :

$$
\begin{aligned}
& {[f(t, x(t))]^{r}=\left[f_{1, r}\left(t, \underline{x}_{r}(t), \bar{x}_{r}(t)\right), f_{2, r}\left(t, \underline{x}_{r}(t), \bar{x}_{r}(t)\right)\right],} \\
& {[g(t, \tau, x(\tau))]^{r}} \\
& \quad=\left[g_{1, r}\left(t, \tau, \underline{x}_{r}(\tau), \bar{x}_{r}(\tau)\right), g_{2, r}\left(t, \tau, \underline{x}_{r}(\tau), \bar{x}_{r}(\tau)\right)\right],
\end{aligned}
$$

are fulfilled by any fuzzy-valued functions obtained from continuous real-valued functions by Zadeh's extension principle and Nguyen theorem. So these conditions are not too restrictive on the mentioned results.

A popular fuzzy real number is the triangular one, which is represented with 3 tuple points $u=\left(u_{L}, u_{C}, u_{R}\right)$, where $u_{C}$ denotes the modal value and the real values $u_{L}$ and $u_{R}$ represent the left and right fuzziness, respectively. This representation is interpreted as membership function $u(s)$ and holds the following conditions: firstly, $u$ is increasing function from $u_{L}$ to $u_{C}$; secondly, $u$ is decreasing function from $u_{C}$ to $u_{R}$; thirdly, $u_{L}<u_{C}<u_{R}$, where

$$
u(s)= \begin{cases}\frac{s-u_{L}}{u_{C}-u_{L}}, & u_{L} \leq s \leq u_{C} \\ \frac{u_{R}-s}{u_{R}-u_{C}}, & u_{C} \leq s \leq u_{R} \\ 0, & u_{L}>s, s>u_{R}\end{cases}
$$

Here, $[u]^{r}=\left[\underline{u}_{r}, \bar{u}_{r}\right]=\left[u_{L}+\left(u_{C}-u_{L}\right) r, u_{R}-\left(u_{R}-u_{C}\right) r\right]$ for each $r \in[0,1]$. We will let $\mathbb{R}_{\mathscr{T}}$ denote the set of triangular fuzzy numbers on $\mathbb{R}$, which is obviously subset of $\mathbb{R}_{\mathscr{F}}$.

Let $x:\left[t_{0}, t_{0}+a\right] \rightarrow \mathbb{R}_{\mathscr{T}}$ be triangular fuzzy-valued function and put $x(t)=\left(x_{L}(t), x_{C}(t), x_{R}(t)\right)$. Then, the representation form of the membership functions of $x(t)$ will be

$$
x(t)(s)= \begin{cases}\frac{s-x_{L}(t)}{x_{C}(t)-x_{L}(t)}, & x_{L}(t) \leq x \leq x_{C}(t), \\ \frac{x_{R}(t)-s}{x_{R}(t)-x_{C}(t)}, & x_{C}(t) \leq x \leq x_{R}(t), \\ 0, & x_{L}(t)>x, x>x_{R}(t) .\end{cases}
$$

Here, $[x(t)]^{r}=\left[\underline{x}_{r}(t), \bar{x}_{r}(t)\right]=\left[x_{L}(t)+\left(x_{C}(t)-x_{L}(t)\right) r, x_{R}(t)-\right.$ $\left.\left(x_{R}(t)-x_{C}(t)\right) r\right]$ for each $r \in[0,1]$.
Theorem 22 (see [40]). Let $x:\left[t_{0}, t_{0}+a\right] \rightarrow \mathbb{R}_{\mathscr{T}}$ be triangular fuzzy-valued function and put $x(t)=\left(x_{L}(t), x_{C}(t), x_{R}(t)\right)$.

(i) if $x$ is (1)-differentiable, then $x_{L}, x_{C}$, and $x_{R}$ are differentiable functions on $\left[t_{0}, t_{0}+a\right]$ and $D_{1} x(t)=$ $\left(x_{L}^{\prime}(t), x_{C}^{\prime}(t), x_{R}^{\prime}(t)\right)$;

(ii) if $x$ is (2)-differentiable, then $x_{L}, x_{C}$, and $x_{R}$ are differentiable functions on $\left[t_{0}, t_{0}+a\right]$ and $D_{2} x(t)=$ $\left(x_{R}^{\prime}(t), x_{C}^{\prime}(t), x_{L}^{\prime}(t)\right)$.

Theorem 23 (see [40]). Let $x:\left[t_{0}, t_{0}+a\right] \rightarrow \mathbb{R}_{\mathscr{T}}$ be continuous triangular fuzzy-valued function and put $x(t)=$ $\left(x_{L}(t), x_{C}(t), x_{R}(t)\right)$. Then $\int_{t_{0}}^{t_{0}+a} x(t) d t$ exist, belonging to $\mathbb{R}_{\mathscr{T}}$, $x_{L}, x_{C}$, and $x_{R}$ are integrable functions on $\left[t_{0}, t_{0}+a\right]$, and $\int_{a}^{b} x(t) d t=\left(\int_{a}^{b} x_{L}(t) d t, \int_{a}^{b} x_{C}(t) d t, \int_{a}^{b} x_{R}(t) d t\right)$.

By using the main properties of Theorems 22 and 23 and by considering the (1)-differentiability concept, if we intend to solve fuzzy VIDE

$$
x^{\prime}(t)=f(t, x(t))+\int_{t_{0}}^{t} g(t, \tau, x(\tau)) d \tau, \quad t_{0} \leq t, \tau \leq t_{0}+a,
$$

subject to the fuzzy initial condition

$$
x\left(t_{0}\right)=\sigma,
$$

where $x(t)=\left(x_{L}(t), x_{C}(t), x_{R}(t)\right)$ and $x\left(t_{0}\right)=\left(\sigma_{L}, \sigma_{C}, \sigma_{R}\right)$, whilst $f:\left[t_{0}, t\right] \times \mathbb{R}_{\mathscr{T}} \rightarrow \mathbb{R}_{\mathscr{T}}$ and $g:\left[t_{0}, t_{0}+a\right]^{2} \times \mathbb{R}_{\mathscr{T}} \rightarrow$ $\mathbb{R}_{\mathscr{T}}$ are continuous triangular fuzzy-valued functions such that

$$
\begin{array}{r}
f(t, x(t))=\left(f_{L}\left(t, x_{L}(t), x_{C}(t), x_{R}(t)\right),\right. \\
f_{C}\left(t, x_{L}(t), x_{C}(t), x_{R}(t)\right), \\
\left.f_{R}\left(t, x_{L}(t), x_{C}(t), x_{R}(t)\right)\right), \\
g(t, \tau, x(\tau))=\left(g_{L}\left(t, \tau, x_{L}(\tau), x_{C}(\tau), x_{R}(\tau)\right),\right. \\
g_{C}\left(t, \tau, x_{L}(\tau), x_{C}(\tau), x_{R}(\tau)\right), \\
\left.g_{R}\left(t, \tau, x_{L}(\tau), x_{C}(\tau), x_{R}(\tau)\right)\right),
\end{array}
$$

then we can translate it into the discretized system of crisp VIDEs as follows:

$$
\begin{aligned}
x_{L}^{\prime}(t)= & f_{L}\left(t, x_{L}(t), x_{C}(t), x_{R}(t)\right) \\
& +\int_{t_{0}}^{t} g_{L}\left(t, \tau, x_{L}(\tau), x_{C}(\tau), x_{R}(\tau)\right) d \tau, \\
x_{C}^{\prime}(t)= & f_{C}\left(t, x_{L}(t), x_{C}(t), x_{R}(t)\right) \\
& +\int_{t_{0}}^{t} g_{C}\left(t, \tau, x_{L}(\tau), x_{C}(\tau), x_{R}(\tau)\right) d \tau, \\
x_{R}^{\prime}(t)= & f_{R}\left(t, x_{L}(t), x_{C}(t), x_{R}(t)\right) \\
& +\int_{t_{0}}^{t} g_{R}\left(t, \tau, x_{L}(\tau), x_{C}(\tau), x_{R}(\tau)\right) d \tau,
\end{aligned}
$$


subject to the crisp initial conditions

$$
x_{L}\left(t_{0}\right)=\sigma_{L}, \quad x_{C}\left(t_{0}\right)=\sigma_{C}, \quad x_{R}\left(t_{0}\right)=\sigma_{R} .
$$

Similarly, in the sense of (2)-differentiability, the fuzzy VIDE (37) can translate into a new discretized form as

$$
\begin{aligned}
x_{L}^{\prime}(t)= & f_{R}\left(t, x_{L}(t), x_{C}(t), x_{R}(t)\right) \\
& +\int_{t_{0}}^{t} g_{R}\left(t, \tau, x_{L}(\tau), x_{C}(\tau), x_{R}(\tau)\right) d \tau, \\
x_{C}^{\prime}(t)= & f_{C}\left(t, x_{L}(t), x_{C}(t), x_{R}(t)\right) \\
& +\int_{t_{0}}^{t} g_{C}\left(t, \tau, x_{L}(\tau), x_{C}(\tau), x_{R}(\tau)\right) d \tau, \\
x_{R}^{\prime}(t)= & f_{L}\left(t, x_{L}(t), x_{C}(t), x_{R}(t)\right) \\
& +\int_{t_{0}}^{t} g_{L}\left(t, \tau, x_{L}(\tau), x_{C}(\tau), x_{R}(\tau)\right) d \tau .
\end{aligned}
$$

Given two triangular fuzzy numbers $x(t)=\left(x_{L}(t), x_{C}(t)\right.$, $\left.x_{R}(t)\right)$ and $y(t)=\left(y_{L}(t), y_{C}(t), y_{R}(t)\right)$, then the Hausdorff distance function between $x(t)$ and $y(t)$ is given by $d_{H}$ : $\mathbb{R}_{\mathscr{T}} \times \mathbb{R}_{\mathscr{T}} \rightarrow \mathbb{R}^{+} \cup\{0\}$ such that $d_{H}(x(t), y(t))=\max \left\{\mid x_{L}(t)-\right.$ $\left.y_{L}(t)|,| x_{C}(t)-y_{C}(t)|,| x_{R}(t)-y_{R}(t) \mid\right\}[9]$.

Theorem 24. Consider the fuzzy VIDEs (37) and (38), where $f:\left[t_{0}, t_{0}+a\right] \times \mathbb{R}_{\mathscr{T}} \rightarrow \mathbb{R}_{\mathscr{T}}$ and $g:\left[t_{0}, t_{0}+a\right]^{2} \times \mathbb{R}_{\mathscr{T}} \rightarrow \mathbb{R}_{\mathscr{T}}$ are triangular fuzzy-valued functions such that

(i) $f(t, x(t))=\left(f_{L}\left(t, x_{L}(t), x_{C}(t), x_{R}(t)\right), f_{C}\left(t, x_{L}(t), x_{C}(t)\right.\right.$, $\left.\left.x_{R}(t)\right), f_{R}\left(t, x_{L}(t), x_{C}(t), x_{R}(t)\right)\right)$, and $g(t, \tau, x(\tau))=$ $\left(g_{L}\left(t, \tau, x_{L}(\tau), x_{C}(\tau), x_{R}(\tau)\right), \quad g_{C}\left(t, \tau, x_{L}(\tau), x_{C}(\tau)\right.\right.$, $\left.\left.x_{R}(\tau)\right), g_{R}\left(t, \tau, x_{L}(\tau), x_{C}(\tau), x_{R}(\tau)\right)\right)$;

(ii) $f_{L}, f_{C}, f_{R}$ and $g_{L}, g_{C}, g_{R}$ are continuous real-valued functions;

(iii) there exists real-finite constants $L_{1}, L_{2}>0$ such that

$$
\begin{aligned}
& \mid f_{L, C, R}\left(t, x_{L}(t), x_{C}(t), x_{R}(t)\right) \\
& -f_{L, C, R}\left(t, y_{L}(t), y_{C}(t), y_{R}(t)\right) \mid \\
& \leq L_{1} \max \left\{\left|x_{L}(t)-y_{L}(t)\right|,\right. \\
& \left.\quad\left|x_{C}(t)-y_{C}(t)\right|,\left|x_{R}(t)-y_{R}(t)\right|\right\}, \\
& \mid g_{L, C, R}\left(t, \tau, x_{L}(\tau), x_{C}(\tau), x_{R}(\tau)\right) \\
& -g_{L, C, R}\left(t, \tau, y_{L}(\tau), y_{C}(\tau), y_{R}(\tau)\right) \mid \\
& \leq L_{2} \max \left\{\left|x_{L}(\tau)-y_{L}(\tau)\right|,\right. \\
& \left.\left|x_{C}(\tau)-y_{C}(\tau)\right|,\left|x_{R}(\tau)-y_{R}(\tau)\right|\right\},
\end{aligned}
$$

for each $t, \tau \in\left[t_{0}, t_{0}+a\right]$ and $x(t), y(t) \in \mathbb{R}_{\mathscr{T}}$. Then, the solutions (one solution for each lateral triangular derivative) of fuzzy VIDEs (37) and (38) are triangular-valued fuzzy function $x(t)=\left(x_{L}(t), x_{C}(t), x_{R}(t)\right):\left[t_{0}, t_{0}+a\right] \times \mathbb{R}_{\mathscr{T}} \rightarrow \mathbb{R}_{\mathscr{T}}$. Whilst, on the other hand, the fuzzy VIDEs (37) and (38) are equivalent to the system of crisp VIDEs (40) and (41) in the sense of (1)differentiability; and, in the sense of (2)-differentiability, the fuzzy VIDEs (37) and (38) and the system of crisp VIDEs (42) and (41) are equivalent.

Proof. It is easy to see that the Lipchitz property of $f_{L}, f_{C}, f_{R}$ and $g_{L}, g_{C}, g_{R}$ implies the continuity of $f$ and $g$ and the Lipchitz property

$$
\begin{gathered}
d_{H}(f(t, x(t)), f(t, y(t))) \leq L_{1} d_{H}((x(t), y(t))), \\
d_{H}(g(t, \tau, x(\tau)), g(t, \tau, y(\tau))) \leq L_{2} d_{H}((x(t), y(t))),
\end{gathered}
$$

for $f$ and $g$, respectively. So, there exists a unique solution, respectively, for both fuzzy VIDEs (37) and (38) and crisp VIDEs (40) and (41). In fact, using Lemma 13, fuzzy VIDEs (37) and (38) is equivalent to a fuzzy integral equation:

$$
x(t)=x_{0}+\int_{t_{0}}^{t} f(s, x(s)) d s+\int_{t_{0}}^{t}\left(\int_{t_{0}}^{s} g(s, \tau, x(\tau)) d \tau\right) d s .
$$

Anyhow, if there exists a solution of (45), since $f(t, x(t))$, $g(t, \tau, x(\tau)) \in \mathbb{R}_{\mathscr{T}}$ and since the integral of a triangularvalued fuzzy function is a triangular fuzzy number, it follows that the unique solution of fuzzy VIDEs (37) and (38) is triangular-valued fuzzy function. On the other aspect as well, it is easy to check that the conditions in Theorem 19 are fulfilled since

$$
\begin{gathered}
\underline{f}_{r}(t, x(t))=f_{L}\left(t, x_{L}(t), x_{C}(t), x_{R}(t)\right) \\
+r\left(f_{C}\left(t, x_{L}(t), x_{C}(t), x_{R}(t)\right)\right. \\
\left.-f_{L}\left(t, x_{L}(t), x_{C}(t), x_{R}(t)\right)\right), \\
\bar{f}_{r}(t, x(t))=f_{R}\left(t, x_{L}(t), x_{C}(t), x_{R}(t)\right) \\
-r\left(f_{R}\left(t, x_{L}(t), x_{C}(t), x_{R}(t)\right)\right. \\
\left.\quad-f_{C}\left(t, x_{L}(t), x_{C}(t), x_{R}(t)\right)\right), \\
\underline{g}_{r}(t, \tau, x(\tau))=g_{L}\left(t, \tau, x_{L}(\tau), x_{C}(\tau), x_{R}(\tau)\right) \\
+r\left(g_{C}\left(t, \tau, x_{L}(\tau), x_{C}(\tau), x_{R}(\tau)\right)\right. \\
\left.\quad-g_{L}\left(t, \tau, x_{L}(\tau), x_{C}(\tau), x_{R}(\tau)\right)\right), \\
\bar{g}_{r}(t, \tau, x(\tau))=g_{R}\left(t, \tau, x_{L}(\tau), x_{C}(\tau), x_{R}(\tau)\right) \\
-r\left(g_{R}\left(t, \tau, x_{L}(\tau), x_{C}(\tau), x_{R}(\tau)\right)\right. \\
\left.\quad-g_{C}\left(t, \tau, x_{L}(\tau), x_{C}(\tau), x_{R}(\tau)\right)\right),
\end{gathered}
$$

where $\underline{x}_{r}(t)=x_{L}(t)+r\left(x_{C}(t)-x_{L}(t)\right)$ and $\bar{x}(t)=x_{R}(t)-$ $r\left(x_{R}(t)-x_{C}(t)\right)$. Finally, the equivalence of fuzzy VIDEs (37) and (38) and crisp VIDEs (40) and (41) follows from Theorems 19, 22, and 23, respectively. So, the proof of the theorem is complete. 
Remark 25. The continuous nonlinear terms $f_{L, C, R}:\left[t_{0}, t_{0}+\right.$ a] $\times \mathbb{R}^{3} \rightarrow \mathbb{R}$ and $g_{L, C, R}:\left[t_{0}, t_{0}+a\right]^{2} \times \mathbb{R}^{3} \rightarrow \mathbb{R}$ are said to satisfy a Lipchitz condition relative to their last argument in the real sense with respect to the specific metric if the conditions (iii) of Theorem 19 hold.

\section{Conclusion}

We know that solving fuzzy IDEs requires appropriate and applicable definitions and theorems to accomplish the mathematical construction. In this paper, we presented and proved the existence and uniqueness of two solutions of fuzzy IDEs of Volterra type based on the Hausdorff distance under the assumption of strongly generalized differentiability for the fuzzy-valued mappings of a real variable whose values are normal, convex, upper semicontinuous, and compactly supported fuzzy sets in $\mathbb{R}$. Whilst, on the other hand, we utilized and proved two characterization theorems for solutions of fuzzy VIDEs which allow us to translate a fuzzy VIDE into a system of crisp VIDEs side by side with some important properties. The proof methodology is based on the assumption of the Lipchitz property for each nonlinear term appearing in the fuzzy VIDE subject to the specific metric used. An efficient computational algorithm is provided to guarantee the procedure and to confirm the performance of the proposed approach. The analogous problem for higherorder fuzzy VIDEs equations is an important subject for future study in this field.

\section{Conflict of Interests}

The authors declare no conflict of interests.

\section{Acknowledgment}

This project was funded by the deanship of scientific research of KAU under Grant no. 28-130-35-HiCi. The authors, therefore, acknowledge the technical and financial support of KAU.

\section{References}

[1] V. Lakshmikantham, K. N. Murty, and J. Turner, “Two-point boundary value problems associated with non-linear fuzzy differential equations," Mathematical Inequalities and Applications, vol. 4, no. 4, pp. 527-533, 2001.

[2] D. O’Regan, V. Lakshmikantham, and J. J. Nieto, "Initial and boundary value problems for fuzzy differential equations," Nonlinear Analysis: Theory, Methods \& Applications, vol. 54, no. 3, pp. 405-415, 2003.

[3] E. Hüllermeier, "An approach to modelling and simulation of uncertain dynamical systems," International Journal of Uncertainty, Fuzziness and Knowlege-Based Systems, vol. 5, no. 2, pp. 117-137, 1997.

[4] D. Li, M. Chen, and X. Xue, "Two-point boundary value problems of uncertain dynamical systems," Fuzzy Sets and Systems, vol. 179, no. 1, pp. 50-61, 2011.

[5] N. Gasilov, Ş. E. Amrahov, and A. G. Fatullayev, "Solution of linear differential equations with fuzzy boundary values," Fuzzy Sets and Systems, vol. 257, pp. 169-183, 2014.
[6] X. Guo, D. Shang, and X. Lu, "Fuzzy approximate solutions of second-order fuzzy linear boundary value problems," Boundary Value Problems, vol. 2013, article 212, 2013.

[7] B. Bede and S. G. Gal, "Almost periodic fuzzy-number-valued functions," Fuzzy Sets and Systems, vol. 147, no. 3, pp. 385-403, 2004.

[8] B. Bede and S. G. Gal, "Generalizations of the differentiability of fuzzy-number-valued functions with applications to fuzzy differential equations," Fuzzy Sets and Systems, vol. 151, no. 3, pp. 581-599, 2005.

[9] B. Bede, "A note on "two-point boundary value problems associated with non-linear fuzzy differential equations'”' Fuzzy Sets and Systems, vol. 157, no. 7, pp. 986-989, 2006.

[10] Y. Chalco-Cano and H. Román-Flores, "On new solutions of fuzzy differential equations," Chaos, Solitons and Fractals, vol. 38, no. 1, pp. 112-119, 2008.

[11] A. Khastan and J. J. Nieto, "A boundary value problem for second order fuzzy differential equations," Nonlinear Analysis: Theory, Methods \& Applications, vol. 72, no. 9-10, pp. 3583-3593, 2010.

[12] J. J. Nieto, A. Khastan, and K. Ivaz, "Numerical solution of fuzzy differential equations under generalized differentiability," Nonlinear Analysis: Hybrid Systems, vol. 3, no. 4, pp. 700-707, 2009.

[13] O. Abu Arqub, "Series solution of fuzzy differential equations under strongly generalized differentiability," Journal of Advanced Research in Applied Mathematics, vol. 5, no. 1, pp. 3152, 2013.

[14] O. Abu-Arqub, A. El-Ajou, S. Momani, and N. Shawagfeh, "Analytical solutions of fuzzy initial value problems by HAM," Applied Mathematics and Information Sciences, vol. 7, no. 5, pp. 1903-1919, 2013.

[15] P. Balasubramaniam and S. Muralisankar, "Existence and uniqueness of fuzzy solution for the nonlinear fuzzy integrodifferential equations," Applied Mathematics Letters, vol. 14, no. 4, pp. 455-462, 2001.

[16] J. Y. Park and J. U. Jeong, "On existence and uniqueness of solutions of fuzzy integrodifferential equations," Indian Journal of Pure and Applied Mathematics, vol. 34, no. 10, pp. 1503-1512, 2003.

[17] J. S. Park and Y. C. Kwun, "Controllability of the nonlinear neutral fuzzy integro-differential equation on $E_{N}^{n}$," Far East Journal of Mathematical Sciences, vol. 19, pp. 11-24, 2005.

[18] P. Prakash and V. Kalaiselvi, "Fuzzy volterra integral equations with infinite delay," Tamkang Journal of Mathematics, vol. 40, no. 1, pp. 19-29, 2009.

[19] S. Hajighasemi, T. Allahviranloo, M. Khezerloo, M. Khorasany, and S. Salahshour, "Existence and uniqueness of solutions of fuzzy Volterra integro-differential equations," Communications in Computer and Information Science, vol. 81, pp. 491-500, 2010.

[20] H. R. Rahimi, M. Khezerloo, and S. Khezerloo, "Approximating the fuzzy solution of the non-linear fuzzy Volterra integrodifferential equation using fixed point theorems," International Journal of Industrial Mathematics, vol. 3, pp. 227-236, 2011.

[21] R. Alikhani, F. Bahrami, and A. Jabbari, "Existence of global solutions to nonlinear fuzzy Volterra integro-differential equations," Nonlinear Analysis: Theory, Methods and Applications, vol. 75, no. 4, pp. 1810-1821, 2012.

[22] T. Allahviranloo, M. Khezerloo, O. Sedaghatfar, and S. Salahshour, "Toward the existence and uniqueness of solutions of second-order fuzzy volterra integro-differential equations 
with fuzzy kernel," Neural Computing and Applications, vol. 22, no. 1, pp. 133-141, 2013.

[23] O. Abu Arqub, "An iterative method for solving fourth-order boundary value problems of mixed type integro-differential equations," Journal of Computational Analysis and Applications. In press.

[24] O. Abu Arqub and M. Al-Smadi, "Numerical algorithm for solving two-point, second-order periodic boundary value problems for mixed integro-differential equations," Applied Mathematics and Computation, vol. 243, pp. 911-922, 2014.

[25] B. Bede, "Note on "Numerical solutions of fuzzy differential equations by predictor-corrector method'”, Information Sciences, vol. 178, no. 7, pp. 1917-1922, 2008.

[26] S. Pederson and M. Sambandham, "Numerical solution of hybrid fuzzy differential equation IVPs by a characterization theorem," Information Sciences, vol. 179, no. 3, pp. 319-328, 2009.

[27] O. Kaleva, "Fuzzy differential equations," Fuzzy Sets and Systems, vol. 24, no. 3, pp. 301-317, 1987.

[28] R. Goetschel Jr. and W. Voxman, "Elementary fuzzy calculus," Fuzzy Sets and Systems, vol. 18, no. 1, pp. 31-43, 1986.

[29] M. L. Puri and D. A. Ralescu, "Fuzzy random variables," Journal of Mathematical Analysis and Applications, vol. 114, no. 2, pp. 409-422, 1986.

[30] M. L. Puri and D. A. Ralescu, "Differentials of fuzzy functions," Journal of Mathematical Analysis and Applications, vol. 91, no. 2, pp. 552-558, 1983.

[31] P. V. Subrahmanyam and S. K. Sudarsanam, "On some fuzzy functional equations," Fuzzy Sets and Systems, vol. 64, no. 3, pp. 333-338, 1994.

[32] C. Wu and Z. Gong, "On Henstock integral of fuzzy-numbervalued functions (I)," Fuzzy Sets and Systems, vol. 120, no. 2, pp. 523-532, 2001.

[33] Y. K. Kim and B. M. Ghil, "Integrals of fuzzy-number-valued functions," Fuzzy Sets and Systems, vol. 86, no. 2, pp. 213-222, 1997.

[34] S. Seikkala, "On the fuzzy initial value problem," Fuzzy Sets and Systems, vol. 24, no. 3, pp. 319-330, 1987.

[35] H. T. Nguyen, "A note on the extension principle for fuzzy sets," Journal of Mathematical Analysis and Applications, vol. 64, no. 2, pp. 369-380, 1978.

[36] H. Román-Flores, L. C. Barros, and R. C. Bassanezi, "A note on Zadeh's extensions," Fuzzy Sets and Systems, vol. 117, no. 3, pp. 327-331, 2001.

[37] R. C. Bassanezi, L. C. de Barros, and P. A. Tonelli, "Attractors and asymptotic stability for fuzzy dynamical systems," Fuzzy Sets and Systems, vol. 113, no. 3, pp. 473-483, 2000.

[38] D. N. Georgiou, J. J. Nieto, and R. Rodríguez-López, "Initial value problems for higher-order fuzzy differential equations," Nonlinear Analysis, Theory, Methods and Applications, vol. 63, no. 4, pp. 587-600, 2005.

[39] S. C. Malik and S. Arora, Mathematical Analysis, Wiley Eastern, New Delhi, India, 2nd edition, 1991.

[40] B. Bede, T. G. Bhaskar, and V. Lakshmikantham, "Perspectives of fuzzy initial value problems," Communications in Applied Analysis, vol. 11, no. 3-4, pp. 339-358, 2007. 


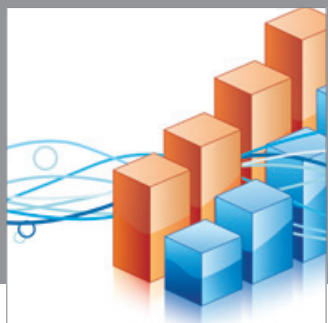

Advances in

Operations Research

mansans

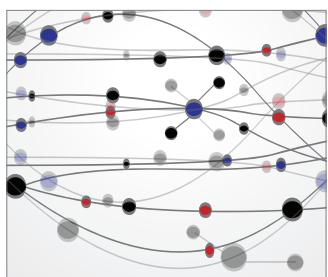

The Scientific World Journal
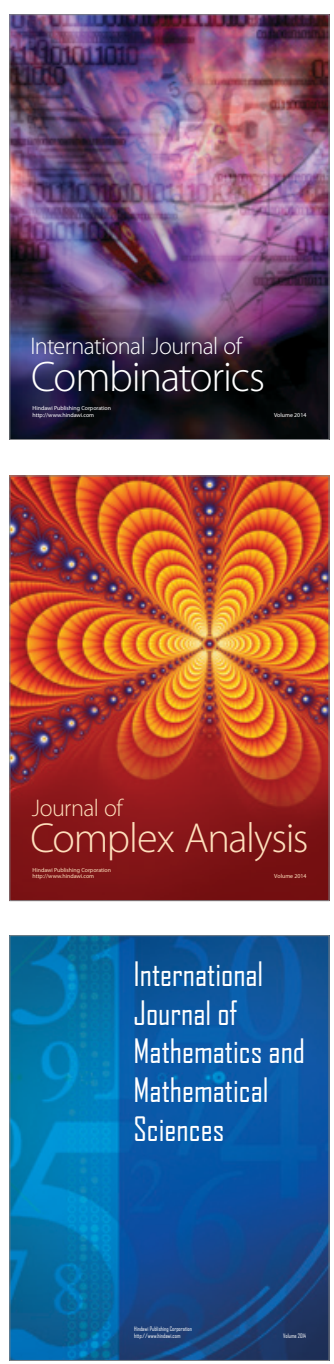
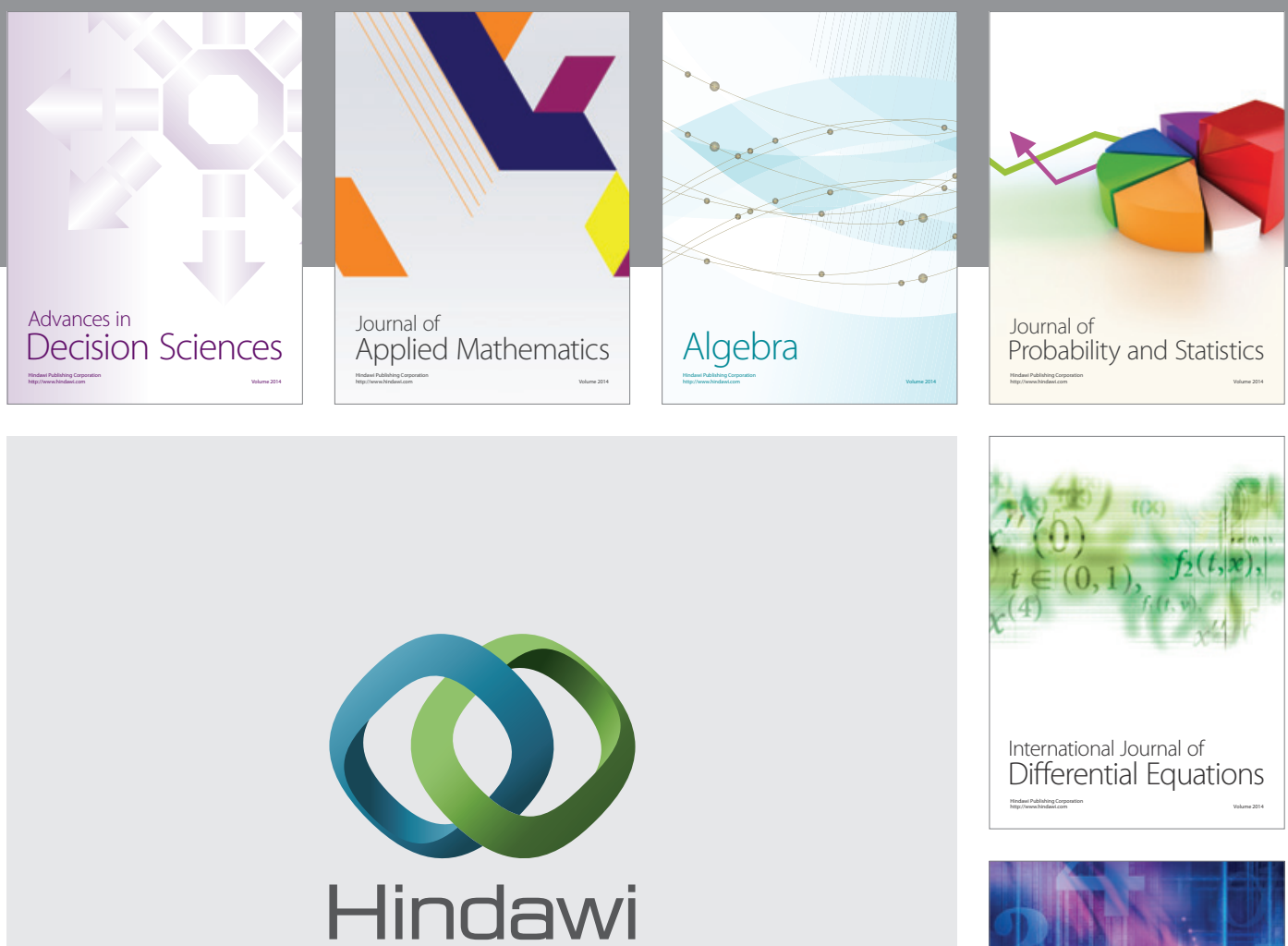

Submit your manuscripts at http://www.hindawi.com
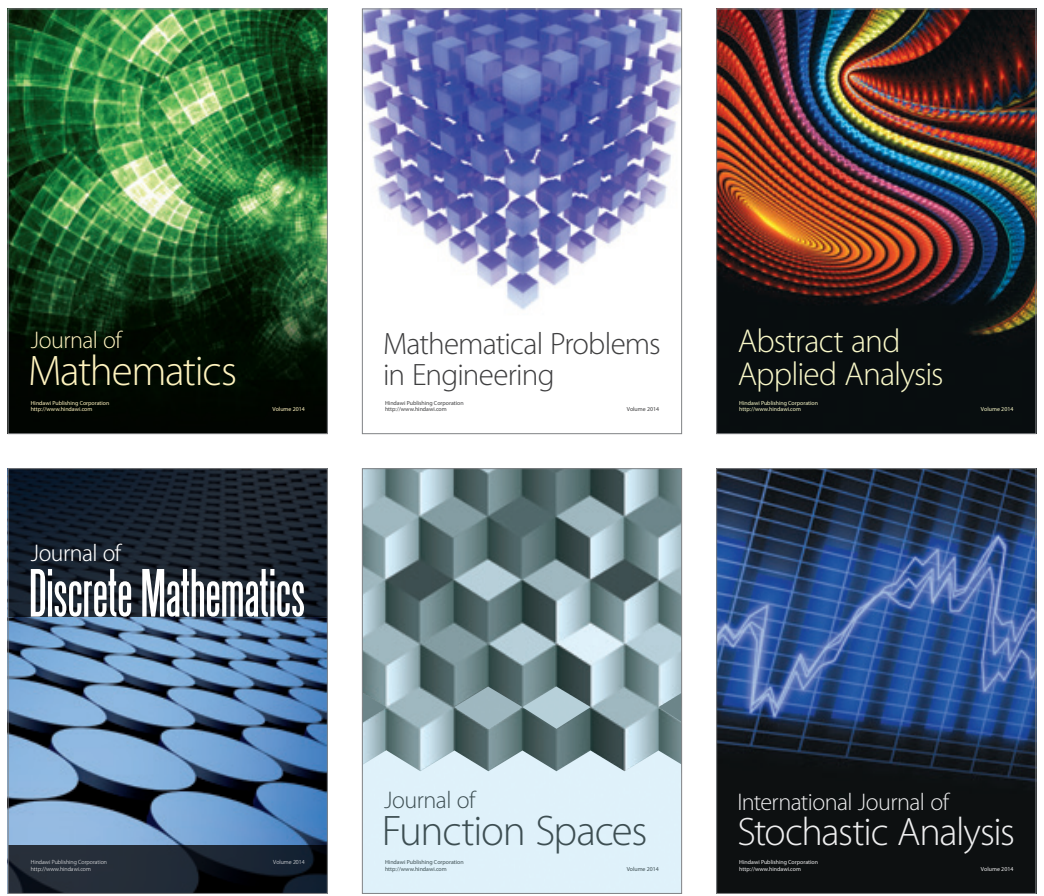

Journal of

Function Spaces

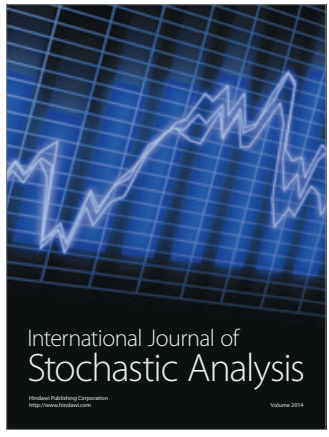

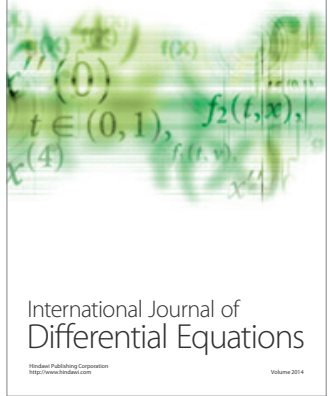
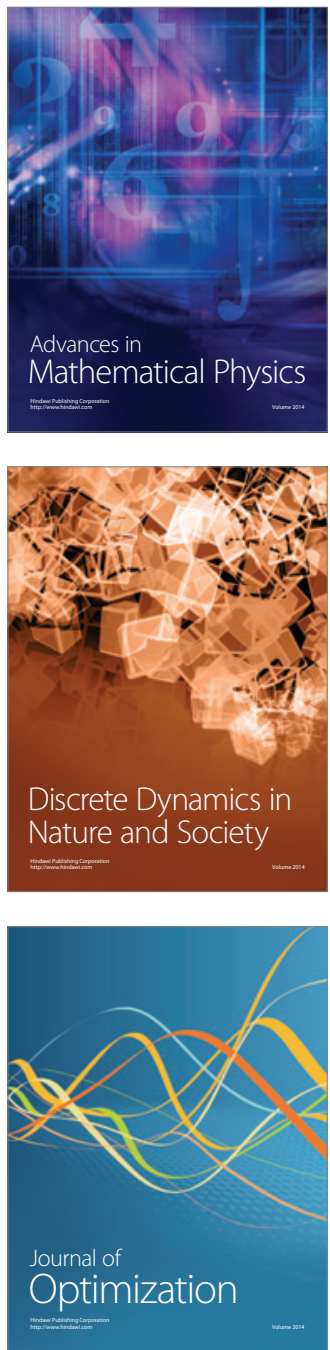